\title{
The ring of differential operators on forms in noncommutative calculus
}

\author{
Dmitri Tamarkin and Boris Tsygan
}

\section{Contents}

1. Introduction

2. Statement of the main theorem

2.1. Gerstenhaber algebras

2.2. Enveloping algebra of a Gerstenhaber algebra

2.3. The Hochschild cochain complex

2.4. The Gerstenhaber algebra $\mathcal{V}^{\bullet}(A)$

2.5. Hochschild chains

2.6. The $A_{\infty}$ algebra $C_{\bullet}\left(C^{\bullet}(A)\right)$

2.7. Statement of the main theorem

3. Sketch of the proof of the main theorem

3.1. Operads $G, G_{\infty}, G_{\text {alg }}, G_{\text {geom }}$

3.2. Calculi

3.3. Pairings between chains and cochains

3.4. The calculus $\operatorname{Calc}(A)$

3.5. Two-colored operads

3.6. Two-colored operads Calc, Calc $_{\infty}$, Calc geom $_{\text {, }}$ Calc alg $_{\text {al }}$

3.7. Enveloping algebra of an algebra over a two-colored operad

3.8. The $A_{\infty}$ module structure on Hochschild chains

3.9. The algebra $Y_{\text {Calc alg }_{\text {alg }}^{0}}\left(C^{\bullet}(A)\right)$ and the Hochschild complex

3.10. The algebra $Y_{\text {Calc }_{\infty}^{0}}\left(C^{\bullet}(A)\right)$ and the Hochschild complex

3.11. Another proof of quasi-isomorphicity of the map (3.11)

3.12. (3.10) is a quasi-isomorphism

3.13. The algebra $Y_{\mathrm{Calc}_{\infty}}\left(C^{\bullet}(A)\right)$ and the cyclic complex

4. Appendix. Hochschild-Gerstenhaber homology

4.1. Introductory remarks

4.2. Hochschild-Gerstenhaber homology vs Hochschild homology

References

2000 Mathematics Subject Classification. 16E40.

Key words and phrases. Hochschild cohomology, Gerstenhaber algebras, operads.

(C)2005 American Mathematical Society 


\section{Introduction}

Many standard geometric objects associated to a manifold $M$ can be defined in terms of the algebra $A$ of functions on $M$. Such definitions can be often made in a manner that makes sense for any associative algebra $A$, commutative or not. The study and applications of these generalized geometric constructions is the subject of noncommutative geometry $[\mathbf{C}],[\mathbf{M}]$.

For example, a vector field on a smooth manifold $M$ can be viewed as a derivation of the algebra $A=C^{\infty}(M)$. If we require such derivations to be local, i.e. to preserve supports, then the Lie algebra of all such derivations is precisely the Lie algebra $\operatorname{Vect}(M)$ of vector fields. One can say that the noncommutative version of $\operatorname{Vect}(M)$ is $\operatorname{Der}(A)$, the Lie algebra of derivations of $A$. Depending on the nature of $A$, one can impose on derivations some conditions like locality, continuity, etc.

Now let us try to define in a similar way the algebra $\mathcal{V}^{\bullet}(M)$ of multivector fields on $M$. The space of multivector fields has a structure of a Gerstenhaber algebra. In other words, it is a graded commutative associative algebra, i.e. the multiplication satisfies

$$
b a=(-1)^{|a||b|} a b ;
$$

$\mathcal{V}^{\bullet}[1]$ is a graded Lie algebra, i.e.

$$
[b, a]=-(-1)^{(|a|-1)(|b|-1)}[a, b]
$$

and

$$
[a,[b, c]]=[[a, b], c]+(-1)^{(|a|-1)(|b|-1)}[b,[a, c]] ;
$$

and the two operations satisfy the Leibnitz identity

$$
[a, b c]=[a, b] c+(-1)^{(|a|-1)|b|} b[a, c]
$$

(cf. $[\mathbf{G}])$.

Throughout the paper, for a complex $\mathcal{V}^{\bullet}$ with differential $d, \mathcal{V}[1]^{k}=\mathcal{V}^{k+1}$ is the complex with the differential $-d$; the ground ring $k$ will be of characteristic zero. For any associative algebra $A$, one can construct a Gerstenhaber algebra $[\mathbf{G}]$; the underlying space of this algebra is the Hochschild cohomology of $A$. We denote it by $H^{\bullet}(A, A)$ or simply by $H^{\bullet}(A)$. It was essentially proven in [HKR] that, when $A=C^{\infty}(M)$, this Gerstenhaber algebra becomes $\mathcal{V}^{\bullet}(M)$ if one understands the Hochschild cohomology properly. More precisely, $H^{\bullet}(A, A)$ for $A=C^{\infty}(M)$ is the cohomology of the complex of Hochschild cochains given by multi-differential expressions.

The problem with the above construction is that the corresponding algebra shrinks considerably as soon as $A$ becomes noncommutative. Indeed, $H^{0}(A, A)$ is the center of $A$, and $H^{1}(A, A)=\operatorname{Der}^{\text {out }}(A)$. So, for example, $H^{0}=\mathbb{C}$ and $H^{1}=0$ for such an important algebra as $A=D\left(\mathbb{R}^{n}\right)$, the ring of differential operators on $\mathbb{R}^{n}$. (In fact for this algebra $H^{i}=0$ for all $i>0$ ).

The problem of constructing a noncommutative analog of the algebra of multivector fields cannot be very easy because the algebra $A$ of "zero-fields" is noncommutative. Consider, for example, the standard Hochschild cochain complex $C^{\bullet}(A, A)$ (see $\left.\S 2.3\right)$. We denote it also by $C^{\bullet}(A)$. It is well known that $C^{\bullet}(A)[1]$ carries a bracket (called the Gerstenhaber bracket) which makes it a dg (differential graded) Lie algebra; $C^{\bullet}(A)$ carries the cup product which makes it a dg associative algebra; at the level of cohomology these two operations induce the standard 
Gerstenhaber algebra structure on $H^{\bullet}(A)$. At the cochain level, however, the associative algebra $C^{\bullet}(A)$ is not commutative (it contains $A=C^{0}$ as a subalgebra).

A solution to this problem was proposed in $[\mathbf{T}]$. It was shown there that the Gerstenhaber bracket and the cup product on $C^{\bullet}(A)$ are part of a much richer algebraic structure, namely that of a $G_{\infty}$ algebra whose underlying $L_{\infty}$ structure is given by the Gerstenhaber bracket (cf. Theorem 2.1.2 of the present paper). There are two equivalent ways to say that a complex $\mathcal{C}^{\bullet}$ is a $G_{\infty}$ algebra. One can define a $G_{\infty}$ structure on $\mathcal{C}^{\bullet}$ explicitly in terms of some multilinear operations on $\mathcal{C}^{\bullet}$, subject to some quadratic relations. Or, equivalently, one can say that there is a differential graded Gerstenhaber algebra $\mathcal{V}^{\bullet}$, quasi-isomorphic to $\mathcal{C}^{\bullet}$ as a complex. Applying this to $C^{\bullet}(A)$, one gets a a dg Gerstenhaber algebra $\mathcal{V}^{\bullet}(A)$ together with a quasi-isomorphism $\mathcal{V}^{\bullet}(A)[1] \rightarrow C^{\bullet}(A)[1]$ of dg Lie algebras. The above quasiisomorphism identifies the cohomology of the complex $\mathcal{V}^{\bullet}(A)$ with $H^{\bullet}(A)$, and this identification is a Gerstenhaber algebra isomorphism. If $A=C^{\infty}(M)$, then the dg Gerstenhaber algebra $\mathcal{V}^{\bullet}(A)$ is quasi-isomorphic to the dg Gerstenhaber algebra $\mathcal{V}^{\bullet}(M)$, the algebra of multivector fields on $M$ with zero differential. (In particular one gets a chain of quasi-isomorphisms of dg Lie algebras

$$
C^{\bullet}(A)[1] \leftarrow \mathcal{V}^{\bullet}(A)[1] \rightarrow \mathcal{V}^{\bullet}(M)[1]
$$

which implies the formality theorem of Kontsevich $[\mathbf{K}]$ ).

The Gerstenhaber algebra $\mathcal{V}^{\bullet}(A)$ is given by a standard tensor construction independent of anything but the vector space $A$. The difficult part is to construct the differential on $\mathcal{V}^{\bullet}(A)$. It is given by a universal formula involving the product on $A$ and some universal coefficients. For these coefficients there seems to be no canonical choice; one can define them if one chooses a Drinfeld associator $[\mathbf{D}]$.

An alternative way to formulate the theorem from $[\mathbf{T}]$ is to say that $C^{\bullet}(A)$ is a $G_{\infty}$ algebra. The notion of a $G_{\infty}$ algebra was introduced in $[\mathbf{G J}]$. By definition, a complex $C^{\bullet}$ is a $G_{\infty}$ algebra if it carries multi-linear operations

$$
m_{k_{1}, \ldots, k_{n}}:\left(C^{\bullet}\right)^{\otimes\left(k_{1}+\ldots+k_{n}\right)} \rightarrow C^{\bullet}
$$

for every $n>0, k_{1}, \ldots, k_{n}>0$; these operations are assumed to satisfy certain symmetry conditions under permutations and certain quadratic equations (which amount to the Maurer-Cartan equation in a certain dg Lie algebra).

For the Hochschild complex $C^{\bullet}(A), m_{1}$ is the differential, $m_{1,1}$ is the Gerstenhaber bracket, and $m_{2}$ is the symmetrized cup product. The higher operations are defined by universal formulas involving the product in $A$ and some coefficients. The choice of these coefficients depends on a choice of a Drinfeld associator.

One can see from this discussion how difficult, inexplicit, and non-canonical the construction of the dg Gerstenhaber algebra $\mathcal{V}^{\bullet}(A)$ is. In view of applications to index theory and other topics, it is natural to ask whether this algebra has some features that are explicit and canonical. For example, as a dg Lie algebra, $\mathcal{V}^{\bullet}(A)[1]$ is quasi-isomorphic to $C^{\bullet}(A)[1]$.

In this paper we propose an answer which has a clear geometric meaning. For any Gerstenhaber algebra $\mathcal{A}^{\bullet}$, one can define an enveloping algebra $Y\left(\mathcal{A}^{\bullet}\right)$, which is a graded associative algebra equipped with a differential $d$. If $\mathcal{A}^{\bullet}=\mathcal{V}^{\bullet}(M)$, the algebra of multivector fields on $M$, then $Y\left(\mathcal{A}^{\bullet}\right)=D\left(\Omega^{\bullet}(M)\right)$, the algebra of differential operators on differential forms on $M$. The differential $d$ acts by commuting an operator with the De Rham differential. 
For a $\operatorname{dg}$ Gerstenhaber algebra $\left(\mathcal{A}^{\bullet}, \delta\right), Y\left(\mathcal{A}^{\bullet}\right)$ inherits a differential which we still denote by $\delta$. By a dg algebra $Y\left(\mathcal{A}^{\bullet}\right)$ we always mean $\left(Y\left(\mathcal{A}^{\bullet}\right), \delta\right)$ (the differential $d$ being ignored).

The first new result of this paper is Theorem 2.6.1. We construct an explicit canonical $A_{\infty}$ algebra whose underlying complex is the Hochschild chain complex $C_{\bullet}\left(C^{\bullet}(A)\right)$ of the dg associative algebra $C^{\bullet}(A)$. (Here, the dg algebra structure on $C^{\bullet}(A)$ is given by the differential and the cup product). In other words, one can construct canonically a $\mathrm{dg}$ associative algebra $\mathrm{D}(A)$, together with a quasiisomorphism of complexes $\mathrm{D}(A) \rightarrow C_{\bullet}\left(C^{\bullet}(A)\right)$. We show (Theorem 2.7.1) that there is an $A_{\infty}$ quasi-isomorphism $Y\left(\mathcal{V}^{\bullet}(A)\right) \rightarrow C_{\bullet}\left(C^{\bullet}(A)\right)$. One can interpret that as a dg algebra quasi-isomorphism of dg algebras $\tilde{Y} \rightarrow D(A)$ where $\tilde{Y}$ is a canonically constructed dg algebra quasi-isomorphic to $Y\left(\mathcal{V}^{\bullet}(A)\right)$.

The above discussion does not take into account the differential $d$ on $Y\left(\mathcal{V}^{\bullet}(A)\right)$. To include $d$ into the picture, note that the $A_{\infty}$ structure on $C_{\bullet}\left(C^{\bullet}(A)\right)$ can be extended to $C_{\bullet}\left(C^{\bullet}(A)\right)[[u]]$, the negative cyclic complex of the $\operatorname{dg}$ algebra $C^{\bullet}(A)$. We show that there is an $A_{\infty}$ quasi-isomorphism

$$
\left(Y\left(\mathcal{V}^{\bullet}(A)\right)[[u]], \delta+u d\right) \rightarrow C_{\bullet}\left(C^{\bullet}(A)\right)[[u]]
$$

Here $\delta$ is the differential on $Y\left(\mathcal{V}^{\bullet}(A)\right)$ induced by the differential on $\mathcal{V}^{\bullet}(A)$.

In other words one can say that the cyclic differential $B$ on the Hochschild complex extends to an $A_{\infty}$ derivation of $C_{\bullet}\left(C^{\bullet}(A)\right)$ (or, if one prefers, to a derivation of $\mathrm{D}(A))$. This derivation is intertwined with the differential $d$ on $Y\left(\mathcal{V}^{\bullet}(A)\right)$.

An extensive sketch of the proof of the main theorem 2.7.1 is given in section 3. A complete proof will be given in a more detailed exposition.

Let us give one example of computing the cohomology ring of the algebra $C_{\bullet}\left(C^{\bullet}(A)\right)$ for a noncommutative algebra $A$. Let $M$ be a symplectic manifold and $A=\left(C^{\infty}(M)[[h]], *\right)$ its deformation quantization ([BFFLS $\left.]\right)$. The following is contained in $[\mathbf{N T}]$.

THEOREM 1.0.1. If $M$ is simply connected, then the cohomology ring of the algebra $C_{\bullet}\left(C^{\bullet}(A)\right)$ is isomorphic to $H^{\bullet}\left(M^{S^{1}}\right)[[h]]$ where $M^{S^{1}}$ is the free loop space of $M$.

Let us say a few words about the $A_{\infty}$ algebras $C_{\bullet}\left(C^{\bullet}(A)\right)$ and $C_{\bullet}\left(C^{\bullet}(A)\right)[[u]]$. The binary products in these algebras were first introduced in $[\mathbf{N T}]$. They were applied to index theorems in $[\mathbf{B N T}]$ and in $[\mathbf{N T 1}]$. In fact their existence and properties were indications that the Gerstenhaber algebra $\mathcal{V}^{\bullet}(A)$ might exist (cf., for example, Theorem 4.3 from $[\mathbf{N T}])$. Some work on the higher operations in $C \bullet\left(C^{\bullet}(A)\right)$ was done in $[\mathbf{M a}]$.

In fact one constructs both a $G_{\infty}$ structure on $C^{\bullet}$ and a canonical $A_{\infty}$ structure on $C_{\bullet}\left(C^{\bullet}\right)[[u]]$ where $C^{\bullet}$ is a dg algebra of a special type, namely a brace algebra. An interpretation of the algebra $C_{\bullet}\left(C^{\bullet}\right)[[u]]$ which is more invariant than ours is given in $[\mathbf{K h}]$. When $C^{\bullet}$ is commutative, one gets the standard shuffle product on Hochschild chains, extending to the $A_{\infty}$ product of Getzler-Jones on negative cyclic chains.

It would be interesting to recover the Gerstenhaber algebra $\mathcal{V}^{\bullet}(A)$ from a less subtle associative algebra $Y\left(\mathcal{V}^{\bullet}(A)\right)$. Note that $Y\left(\mathcal{V}^{\bullet}(A)\right)$ has an increasing filtration $F_{n}, n \geq 0$, such that:

- $F_{0}=\mathcal{V}^{\bullet}(A) ; F_{m} F_{n} \subset F_{m+n}$;

- $\operatorname{gr}_{F} Y$ is graded commutative; 
- $d F_{n} \subset F_{n+1}$

Given any algebra with such filtration, one can recover the Gerstenhaber algebra structure on $F_{0}$ : the product comes from the one on $Y$, and the bracket is the derived bracket $[a, d b]$. It would be interesting to understand how to construct directly a family of filtrations on the canonical algebra $Y$, indexed by Drinfeld associators.

Let us finish by outlining a few possible areas of study in the future.

1. Connes-Moscovici type index theorems. Computations similar to those in $C_{\bullet}\left(C^{\bullet}(A)\right)[[u]]$ are used in $[\mathbf{C M}],[\mathbf{C 1}]$. It would be very interesting to find a unified framework for both approaches. In particular, the symmetry group acting on the space of possible choices of $\mathcal{V}^{\bullet}(A)$ is the Grothendieck-Teichmuller group which is closely related to $\operatorname{Gal}(\overline{\mathbb{Q}} / \mathbb{Q})$. In $[\mathbf{C M}]$ and $[\mathbf{C 1}]$, the symmetry with respect to the renormalization group was used. Part of Connes' program of noncommutative geometry is to unite the renormalization group and $\operatorname{Gal}(\overline{\mathbb{Q}} / \mathbb{Q})$ (the second author thanks Alain Connes for helpful comments on this subject).

2. Quantum cohomology and the Fukaya category. Starting from a compact symplectic manifold $M$, one can construct the quantum cohomology ring $H Q^{\bullet}(M)$ (cf., for example, [MDS] or $[\mathbf{K M}]$ ) and the $A_{\infty}$ category $\mathcal{F}(M)$ (cf. [F1]). Conjecturally, the former is the Hochschild cohomology of the latter (cf. [Sei]).

One can generalize the construction of the algebra $Y\left(C^{\bullet}(A)\right)$, or in fact the construction of both sides in Theorem 2.7.1, and replace an algebra $A$ by an $A_{\infty}$ category $\mathcal{F}$. Thus, one gets an associative dg algebra

$$
Y(M)=Y\left(C^{\bullet}(\mathcal{F}(M))\right)
$$

Its cohomology algebra, possibly noncommutative, we denote by $\mathcal{H} Q^{\bullet}(M)$. If the conjecture from $[\mathbf{S e i}]$ is true, then we get a morphism of algebras

$$
H Q^{\bullet}(M) \rightarrow \mathcal{H} Q^{\bullet}(M)
$$

The right hand side should be closely related to the cohomology of the free loop space of $M$, as suggested by Theorem 1.0.1.

3. Topological string theory of Chas-Sullivan. It is strongly believed that, for an oriented compact manifold $X$, the chain complex $C_{\bullet}\left(X^{S^{1}}\right)$ is a $G_{\infty}$ algebra, even a $\mathrm{BV}_{\infty}$ algebra, cf. $[\mathbf{C S}]$. It would be interesting to study the enveloping algebra $Y\left(\mathcal{V}^{\bullet}(X)\right)$ where $\mathcal{V}^{\bullet}(X)$ is the standard resolution of $C_{\bullet}\left(X^{S^{1}}\right)$. Denote its cohomology algebra by $\mathcal{H}_{\text {loop }}^{\bullet}(X)$. One gets a morphism of algebras

$$
H_{n-\bullet}\left(X^{S^{1}}\right) \rightarrow \mathcal{H}_{\text {loop }}^{\bullet}(X)
$$

The algebra $\mathcal{H}_{\text {loop }}^{\bullet}(X)$, possibly noncommutative, should be related to the double loop space of $X$.

A link between points $\mathbf{2}$ and $\mathbf{3}$ seems to be indicated in [Sei].

Acknowledgements. Both authors' work was partially supported by NSF grants. The authors would like to thank A. Beilinson, P. Bressler, A. Connes, V. Drinfeld, B. Feigin, K. Fukaya, E. Getzler, M. Khalkhali, R. Nest and A. Voronov for stimulating discussions. The second author thanks the Dennisfest organizers for their hospitality and for the wonderful scientific atmosphere. 


\section{Statement of the main theorem}

2.1. Gerstenhaber algebras. Let $k$ be the ground ring of characteristic zero. A Gerstenhaber algebra is a graded space $\mathcal{V}^{\bullet}$ together with

- A graded commutative associative algebra structure on $\mathcal{V}^{\bullet}$;

- a graded Lie algebra structure on $\mathcal{V}^{\bullet+1}$ such that

$$
[a, b c]=[a, b] c+(-1)^{\operatorname{deg}(a) \operatorname{deg}(b)} b[a, c]
$$

EXAmPLE 2.1.1. Let $\mathfrak{g}$ be a Lie algebra. Then

$$
C \bullet(\mathfrak{g})=\wedge \mathfrak{g}
$$

is a Gerstenhaber algebra.

The product is the exterior product, and the bracket is the unique bracket which turns $C_{\bullet}(\mathfrak{g})$ into a Gerstenhaber algebra and which is the Lie bracket on $\mathfrak{g}=\wedge^{1}(\mathfrak{g})$.

EXAMPLE 2.1.2. Let $M$ be a smooth manifold. Then

$$
\mathcal{V}_{M}^{\bullet}=\wedge^{\bullet} T_{M}
$$

is a sheaf of Gerstenhaber algebras.

The product is the exterior product, and the bracket is the Schouten bracket. We denote by $\mathcal{V} \bullet(M)$ the Gerstenhaber algebra of global sections of this sheaf. The previous example is the algebra of left-invariant multivector fields on the Lie group of $\mathfrak{g}$.

2.2. Enveloping algebra of a Gerstenhaber algebra. The following construction is motivated by Example 2.1.2. For a Gerstenhaber algebra $\mathcal{V}^{\bullet}$, let $Y\left(\mathcal{V}^{\bullet}\right)$ be the associative algebra generated by two sets of generators $i_{a}, L_{a}, a \in \mathcal{V}^{\bullet}$, both $i$ and $L$ linear in $a$,

subject to relations

$$
\left|i_{a}\right|=|a| ;\left|L_{a}\right|=|a|-1
$$

$$
\begin{gathered}
i_{a} i_{b}=i_{a b} ; \quad\left[L_{a}, L_{b}\right]=L_{[a, b]} ; \\
{\left[L_{a}, i_{b}\right]=i_{[a, b]} ; \quad L_{a b}=L_{a} i_{b}+(-1)^{|a|} i_{a} L_{b}}
\end{gathered}
$$

The algebra $Y\left(\mathcal{V}^{\bullet}\right)$ is equipped with the differential $d$ of degree one which is defined as a derivation sending $i_{a}$ to $L_{a}$ and $L_{a}$ to zero.

For a smooth manifold $M$ one has a homomorphism

$$
Y\left(\mathcal{V}^{\bullet}(M)\right) \rightarrow \mathrm{D}\left(\Omega^{\bullet}(M)\right)
$$

The right hand side is the algebra of differential operators on differential forms on $M$. It is easy to see that this is in fact an isomorphism.

2.3. The Hochschild cochain complex. Let $A$ be a graded associative algebra with unit 1 over a commutative unital ring $k$ of characteristic zero. A Hochschild $d$-cochain is a linear map $A^{\otimes d} \rightarrow A$. Put, for $d \geq 0$,

$$
C^{d}(A)=C^{d}(A, A)=\operatorname{Hom}_{k}\left(\bar{A}^{\otimes d}, A\right)
$$

where $\bar{A}=A /(k \cdot 1)$ is the quotient linear $k$-space. Elements of $C^{d}(A)$ are called normalized cochains. We prefer to work with normalized cochains because the formulas for pairings between chains and cochains are simpler. 
Put

$$
|D|=(\text { degree of the linear map } D)+d
$$

Put for cochains $D$ and $E$ from $C^{\bullet}(A, A)$

$$
\begin{gathered}
(D \smile E)\left(a_{1}, \ldots, a_{d+e}\right)=(-1)^{|E| \sum_{i \leq d}\left(\left|a_{i}\right|+1\right)} D\left(a_{1}, \ldots, a_{d}\right) \times \\
\times E\left(a_{d+1}, \ldots, a_{d+e}\right) ; \\
(D \circ E)\left(a_{1}, \ldots, a_{d+e-1}\right)=\sum_{j \geq 0}(-1)^{(|E|+1) \sum_{i=1}^{j}\left(\left|a_{i}\right|+1\right)} \\
D\left(a_{1}, \ldots, a_{j}, E\left(a_{j+1}, \ldots, a_{j+e}\right), \ldots\right) ; \\
{[D, E]=D \circ E-(-1)^{(|D|+1)(|E|+1)} E \circ D}
\end{gathered}
$$

These operations define the graded associative algebra $\left(C^{\bullet}(A, A), \smile\right)$ and the graded Lie algebra $\left(C^{\bullet+1}(A, A),[],\right)(\mathrm{cf}$. $[\mathbf{C E}] ;[\mathbf{G}])$. Let

$$
m\left(a_{1}, a_{2}\right)=(-1)^{\operatorname{deg} a_{1}} a_{1} a_{2} ;
$$

this is a 2-cochain of $A$ (not in $C^{2}$ ). Put

$$
\begin{gathered}
\delta D=[m, D] \\
(\delta D)\left(a_{1}, \ldots, a_{d+1}\right)=(-1)^{\left|a_{1}\right||D|+|D|+1} \times \\
\times a_{1} D\left(a_{2}, \ldots, a_{d+1}\right)+ \\
+\sum_{j=1}^{d}(-1)^{|D|+1+\sum_{i=1}^{j}\left(\left|a_{i}\right|+1\right)} D\left(a_{1}, \ldots, a_{j} a_{j+1}, \ldots, a_{d+1}\right) \\
+(-1)^{|D| \sum_{i=1}^{d}\left(\left|a_{i}\right|+1\right)} D\left(a_{1}, \ldots, a_{d}\right) a_{d+1}
\end{gathered}
$$

One has

$$
\begin{gathered}
\delta^{2}=0 ; \quad \delta(D \smile E)=\delta D \smile E+(-1)^{|\operatorname{deg} D|} D \smile \delta E \\
\delta[D, E]=[\delta D, E]+(-1)^{|D|+1}[D, \delta E]
\end{gathered}
$$

$\left(\delta^{2}=0\right.$ follows from $\left.[m, m]=0\right)$.

Thus $C^{\bullet}(A, A)$ becomes a complex; we will denote it also by $C^{\bullet}(A)$. The cohomology of this complex is $H^{\bullet}(A, A)$ or the Hochschild cohomology.

We denote it also by $H^{\bullet}(A)$. The $\smile$ product induces the Yoneda product on $H^{\bullet}(A, A)=\operatorname{Ext}_{A \otimes A^{0}}^{\bullet}(A, A)$. The operation [ , ] is the Gerstenhaber bracket [G].

If $(A, \partial)$ is a differential graded algebra then one can define the differential $\partial$ acting on $C^{\bullet}(A)$ by:

$$
\partial D=[\partial, D]
$$

TheOREm 2.3.1. [G] The cup product and the Gerstenhaber bracket induce a Gerstenhaber algebra structure on $H^{\bullet}(A)$.

For cochains $D$ and $D_{i}$ define a new Hochschild cochain by the following formula of Gerstenhaber $([\mathbf{G}])$ and Getzler $([\mathbf{G} \mathbf{1}])$ :

$$
\begin{gathered}
D_{0}\left\{D_{1}, \ldots, D_{m}\right\}\left(a_{1}, \ldots, a_{n}\right)= \\
=\sum(-1)^{\sum_{k \leq i_{p}}\left(\left|a_{k}\right|+1\right)\left(\left|D_{p}\right|+1\right)} D_{0}\left(a_{1}, \ldots, a_{i_{1}}, D_{1}\left(a_{i_{1}+1}, \ldots\right), \ldots,\right. \\
\left.D_{m}\left(a_{i_{m}+1}, \ldots\right), \ldots\right)
\end{gathered}
$$


Proposition 2.3.2. One has

$$
\begin{aligned}
& \left(D\left\{E_{1}, \ldots, E_{k}\right\}\right)\left\{F_{1}, \ldots, F_{l}\right\}=\sum(-1)^{\sum_{q \leq i_{p}}\left(\left|E_{p}\right|+1\right)\left(\left|F_{q}\right|+1\right)} \times \\
& \quad \times D\left\{F_{1}, \ldots, E_{1}\left\{F_{i_{1}+1}, \ldots,\right\}, \ldots, E_{k}\left\{F_{i_{k}+1}, \ldots,\right\}, \ldots,\right\}
\end{aligned}
$$

The above proposition can be restated as follows. For a cochain $D$ let $D^{(k)}$ be the following $k$-cochain of $C^{\bullet}(A)$ :

$$
D^{(k)}\left(D_{1}, \ldots, D_{k}\right)=D\left\{D_{1}, \ldots, D_{k}\right\}
$$

Proposition 2.3.3. The map

$$
D \mapsto \sum_{k \geq 0} D^{(k)}
$$

is a morphism of differential graded algebras

$$
C^{\bullet}(A) \rightarrow C^{\bullet}\left(C^{\bullet}(A)\right)
$$

2.4. The Gerstenhaber algebra $\mathcal{V}^{\bullet}(A)$. Below is the theorem from $[\mathbf{T}]$. We sketch its proof in 3.1 .

Theorem 2.4.1. For every associative algebra A there exists a $d g$ Gerstenhaber algebra $\mathcal{V}^{\bullet}(A)$ such that:

- There is a quasi-isomorphism of dg Lie algebras

$$
\mathcal{V}^{\bullet}(A)[1] \rightarrow C^{\bullet}(A)[1]
$$

- The above quasi-isomorphism induces an isomorphism of Gerstenhaber algebras

$$
H^{\bullet}\left(\mathcal{V}^{\bullet}(A)\right) \rightarrow H^{\bullet}(A)
$$

where the Gerstenhaber structure on the right hand side is the standard one from 2.3.

- For $A=C^{\infty}(M)$ there is a quasi-isomorphism of $d g$ Gerstenhaber algebras

$$
\mathcal{V}^{\bullet}(A) \rightarrow \mathcal{V}^{\bullet}(M)
$$

2.5. Hochschild chains. Let $A$ be an associative dg algebra with unit 1 over a ground ring $k$. The differential on $A$ is denoted by $\delta$. Recall that by definition

$$
\bar{A}=A /(k \cdot 1)
$$

Set

$$
C_{p}(A, A)=C_{p}(A)=A \otimes \bar{A}^{\otimes p}
$$

Define the differentials $\delta: C_{\bullet}(A) \rightarrow C_{\bullet}(A), b: C_{\bullet}(A) \rightarrow C_{\bullet-1}(A), B: C_{\bullet}(A) \rightarrow$ $C_{\bullet+1}(A)$ as follows.

$$
\begin{gathered}
\delta\left(a_{0} \otimes \cdots \otimes a_{p}\right)=\sum_{i=1}^{p}(-1)^{\sum_{k<i}\left(\left|a_{k}\right|+1\right)+1}\left(a_{0} \otimes \cdots \otimes \delta a_{i} \otimes \cdots \otimes a_{p}\right) \\
b\left(a_{0} \otimes \ldots \otimes a_{p}\right)=\sum_{k=0}^{p-1}(-1)^{\sum_{i=0}^{k}\left(\left|a_{i}\right|+1\right)+1} a_{0} \ldots \otimes a_{k} a_{k+1} \otimes \ldots a_{p} \\
+(-1)^{\left|a_{p}\right|+\left(\left|a_{p}\right|+1\right) \sum_{i=0}^{p-1}\left(\left|a_{i}\right|+1\right)} a_{p} a_{0} \otimes \ldots \otimes a_{p-1}
\end{gathered}
$$




$$
\begin{gathered}
B\left(a_{0} \otimes \ldots \otimes a_{p}\right)=\sum_{k=0}^{p}(-1)^{\sum_{i \leq k}\left(\left|a_{i}\right|+1\right) \sum_{i \geq k}\left(\left|a_{i}\right|+1\right)} 1 \otimes a_{k+1} \otimes \ldots a_{p} \otimes \\
\otimes a_{0} \otimes \ldots \otimes a_{k}
\end{gathered}
$$

The complex $C \bullet(A)$ is the total complex of the double complex with the differential $b+\delta$.

Let $u$ be a formal variable of degree -2 . The complex $\left(C^{\bullet}(A)[[u]], b+\delta+u B\right)$ is called the negative cyclic complex of $A$.

One can define a product

$$
\operatorname{sh}: C^{\bullet}(A) \otimes C^{\bullet}(A) \rightarrow C^{\bullet}(A)
$$

and its extension

$$
\operatorname{sh}+u \operatorname{sh}^{\prime}: C^{\bullet}(A)[[u]] \otimes C^{\bullet}(A)[[u]] \rightarrow C^{\bullet}(A)[[u]]
$$

$[\mathbf{L}]$ by the following explicit formulas:

$$
\left(a_{0} \otimes \ldots \otimes a_{p}\right) \otimes\left(c_{0} \otimes \ldots \otimes c_{q}\right) \stackrel{\text { sh }}{\mapsto} a_{0} c_{0} \otimes \operatorname{sh}_{p q}\left(a_{1}, \ldots, a_{p}, c_{1}, \ldots, c_{q}\right)
$$

where

$$
\operatorname{sh}_{p q}\left(x_{1}, \ldots, x_{p+q}\right)=\sum_{\sigma \in \operatorname{Sh}(p, q)} \operatorname{sgn}(\sigma) x_{\sigma^{-1} 1} \otimes \ldots \otimes x_{\sigma^{-1}(p+q)}
$$

and

$$
\operatorname{Sh}(p, q)=\left\{\sigma \in \Sigma_{p+q} \mid \sigma 1<\ldots<\sigma p ; \sigma(p+1)<\ldots<\sigma(p+q)\right\}
$$

In the graded case, $\operatorname{sgn}(\sigma)$ gets replaced by the sign computed by the following rule: in all transpositions, the parity of $a_{i}$ is equal to $\left|a_{i}\right|+1$ if $i \neq 0$ and $\left|a_{0}\right|$ if $i=0$, and similarly for $c_{i}$. A transposition contributes a product of parities.

$$
\left(a_{0} \otimes \ldots \otimes a_{p}\right) \otimes\left(c_{0} \otimes \ldots \otimes c_{q}\right) \stackrel{\mathrm{sh}^{\prime}}{\mapsto} 1 \otimes \operatorname{sh}_{p+1, q+1}^{\prime}\left(a_{0}, \ldots, a_{p}, c_{0}, \ldots, c_{q}\right)
$$

where

$$
\operatorname{sh}_{p+1, q+1}^{\prime}\left(x_{0}, \ldots, x_{p+q+1}\right)=\sum_{\sigma \in \operatorname{Sh}^{\prime}(p+1, q+1)} \operatorname{sgn}(\sigma) x_{\sigma^{-1} 0} \otimes \ldots \otimes x_{\sigma^{-1}(p+q+1)}
$$

and $\operatorname{Sh}^{\prime}(p+1, q+1)$ is the set of all permutations $\sigma \in \Sigma_{p+q+2}$ such that $\sigma 0<\ldots<$ $\sigma p, \sigma(p+1)<\ldots<\sigma(p+q+1)$, and $\sigma 0<\sigma(p+1)$

2.6. The $A_{\infty}$ algebra $C_{\bullet}\left(C^{\bullet}(A)\right)$. Recall $[\mathbf{L S}],[\mathbf{S t 1}]$ that an $A_{\infty}$ algebra is a graded vector space $\mathcal{C}$ together with a Hochschild cochain $m$ of total degree 1 ,

$$
m=\sum_{n=1}^{\infty} m_{n}
$$

where $m_{n} \in C^{n}(\mathcal{C})$ and

$$
[m, m]=0
$$

Consider the Hochschild cochain complex of a graded algebra $A$ as a differential graded associative algebra $\left(C^{\bullet}(A), \smile, \delta\right)$. Consider the Hochschild chain complex 
of this differential graded algebra. The total differential in this complex is $b+\delta$; the degree of a chain is given by

$$
\left|D_{0} \otimes \ldots \otimes D_{n}\right|=\left|D_{0}\right|+\sum_{i=1}^{n}\left(\left|D_{i}\right|+1\right)
$$

where $D_{i}$ are Hochschild cochains.

The complex $C_{\bullet}\left(C^{\bullet}(A)\right)$ contains the Hochschild cochain complex $C^{\bullet}(A)$ as a subcomplex (of zero-chains) and has the Hochschild chain complex $C_{\bullet}(A)$ as a quotient complex:

$$
C^{\bullet}(A) \stackrel{i}{\longrightarrow} C_{\bullet}\left(C^{\bullet}(A)\right) \stackrel{\pi}{\longrightarrow} C_{\bullet}(A)
$$

(this sequence is not by any means exact). The projection on the right splits if $A$ is commutative. If not, $C_{\bullet}(A)$ is naturally a graded subspace but not a subcomplex.

Theorem 2.6.1. There is an $A_{\infty}$ structure on $C_{\bullet}\left(C^{\bullet}(A)\right)[[u]]$ such that:

- All $m_{n}$ are $k[[u]]$-linear, $(u)$-adically continuous

- $m_{1}=b+\delta+u B$

$$
\text { For } x, y \in C_{\bullet}(A) \text { : }
$$

- $(-1)^{|x|} m_{2}(x, y)=\left(\operatorname{sh}+u \operatorname{sh}^{\prime}\right)(x, y)$

For $D, E \in C^{\bullet}(A)$ :

- $(-1)^{|D|} m_{2}(D, E)=D \smile E$

- $m_{2}(1 \otimes D, 1 \otimes E)+(-1)^{|D||E|} m_{2}(1 \otimes E, 1 \otimes D)=(-1)^{|D|} 1 \otimes[D, E]$

- $m_{2}(D, 1 \otimes E)+(-1)^{(|D|+1)|E|} m_{2}(1 \otimes E, D)=(-1)^{|D|+1}[D, E]$

Here is an explicit description of the above $A_{\infty}$ structure. We define for $n \geq 2$

$$
m_{n}=m_{n}^{(1)}+m_{n}^{(2)}+u m_{n}^{(3)}
$$

where, for

$$
\begin{gathered}
a^{(k)}=D_{0}^{(k)} \otimes \ldots \otimes D_{N_{k}}^{(k)}, \\
m_{n}^{(1)}=0
\end{gathered}
$$

for $n \geq 3$;

$$
\begin{gathered}
m_{2}^{(1)}\left(a^{(1)}, a^{(2)}\right)=(-1)^{\left|a^{(1)}\right|} \sum \pm D_{0}^{(1)} \smile D_{0}^{(2)} \underline{\{\}} \otimes \underline{\ldots \otimes} \\
\otimes D_{1}^{(2)} \underline{\{\}} \otimes \ldots \ldots \otimes D_{N_{2}}^{(2)} \underline{\{\}} \otimes \ldots
\end{gathered}
$$

The space designated by ${ }_{-}$is filled with $D_{1}^{(1)}, \ldots, D_{N_{1}}^{(1)}$ whose order is preserved. The sign rule is as follows: the parity of $D_{j}^{(i)}$ is $\left|D_{j}^{(i)}\right|$ for $j=0$ and $\left|D_{j}^{(i)}\right|+1$ otherwise.

$$
\begin{gathered}
m_{n}^{(2)}\left(a^{(1)}, \ldots, a^{(n)}\right)= \\
=(-1)^{\sum_{i=1}^{n-1}\left|a_{1}\right|+n} \sum \pm D_{N_{n}}^{(n)} \underline{\left\{\ldots, D_{0}^{(1)}, \ldots, D_{0}^{(n-1)}\left\{{ }_{-}\right\}, \ldots\right\}} \smile \\
\smile D_{0}^{(n)}\left\{{ }_{-}\right\} \otimes \ldots \otimes D_{1}^{(n)} \underline{\{\}} \underline{\ldots} \ldots \otimes D_{N_{n}-1}^{(n)} \underline{\{\}} \otimes \ldots
\end{gathered}
$$

The space designated by _ is filled with $D_{i}^{(j)}$ for $j<n$ in such a way that:

- the cyclic order of each group $D_{0}^{(k)}, \ldots, D_{N_{k}}^{(k)}$ is preserved

- $D_{0}^{(1)}, \ldots, D_{0}^{(n-1)}$ are all inside the braces in $D_{N_{n}}^{(n)}\{\}$

- $D_{0}^{(i)}$ is to the left of $D_{0}^{(j)}$ for $i<j$ 
- any cochain $D_{j}^{(i)}$ may contain some of its neighbors on the right inside the braces, provided that all of these neighbors are of the form $D_{q}^{(p)}$ with $p<i$

The parity of $D_{j}^{(i)}$ is $\left|D_{j}^{(i)}\right|$ if $i=n$ and $j=0$; it is $\left|D_{j}^{(i)}\right|+1$ otherwise. Note that the formula for $m_{n}^{(2)}$ gives the Hochschild chain differential $b$ for $n=1$.

Finally, define

$$
m_{n}^{(3)}\left(a^{(1)}, \ldots, a^{(n)}\right)=(-1)^{n+1} \sum \pm 1 \otimes \underline{\ldots \otimes D_{0}^{(0)} \otimes \ldots \otimes D_{0}^{(n)}\{\} \otimes \ldots}
$$

The underlined space is filled with $D_{i}^{(j)}$ in such a way that:

- the cyclic order of each group $D_{0}^{(k)}, \ldots, D_{N_{k}}^{(k)}$ is preserved

- $D_{0}^{(i)}$ is to the left of $D_{0}^{(j)}$ for $i<j$

- any cochain $D_{j}^{(i)}$ may contain some of its neighbors on the right inside the braces, provided that all of these neighbors are of the form $D_{q}^{(p)}$ with $p<i$. The parity of $D_{j}^{(i)}$ is always $\left|D_{j}^{(i)}\right|+1$.

One checks by a direct computation that the above formulas provide an $A_{\infty}$ structure on $C_{\bullet}\left(C^{\bullet}(A)\right)[[u]]$.

REmark 2.6.2. Let $A$ be a commutative algebra. Then $C \bullet(A)[[u]]$ is not only a subcomplex but an $A_{\infty}$ subalgebra of $C_{\bullet}\left(C^{\bullet}(A)\right)[[u]]$. This $A_{\infty}$ structure on $C \cdot(A)[[u]]$ was introduced in $[\mathbf{G J} \mathbf{1}]$.

\subsection{Statement of the main theorem.}

TheOREM 2.7.1. There is a $A_{\infty}$ quasi-isomorphism

$$
Y\left(\mathcal{V}^{\bullet}(A)\right) \rightarrow C_{\bullet}\left(C^{\bullet}(A)\right)
$$

which extends to a $k[[u]]$-linear, $(u)$-adically continuous $A_{\infty}$ quasi-isomorphism

$$
\left(Y\left(\mathcal{V}^{\bullet}(A)\right)[[u]], \delta+u d\right) \rightarrow C_{\bullet}\left(C^{\bullet}(A)\right)[[u]]
$$

\section{Sketch of the proof of the main theorem}

We will start by introducing several operads used in the proof of Theorem 2.1.2. We will then extend the notion of a Gerstenhaber algebra to that of a calculus (3.2). Next we will introduce some basic pairings between Hochschild chains and cochains, extending the definitions from 2.5. Then we will state Theorem 3.4.1 which asserts that the Gerstenhaber algebra $\mathcal{V}^{\bullet}(A)$ can be extended to a calculus $\operatorname{Calc}(A)$.

Next, we will introduce a notion of a two-colored operad which is suitable for working with objects like calculi (3.5). After that we extend the contents of 3.1 by introducing corresponding two-colored operads and stating relations among them (3.6).

Next, we define a notion of the enveloping algebra of an algebra $\mathcal{A}^{\bullet}$ over an operad $\mathcal{O}$, provided that $\mathcal{O}$ is part of a two-colored operad $\mathcal{P}$. This is an associative dg algebra which we denote by $Y_{\mathcal{P}}\left(\mathcal{A}^{\bullet}\right)$. Because of Theorem 3.4.1, one has morphisms of dg algebras

$$
Y_{\mathrm{Calc}_{\mathrm{alg}}^{0}}\left(\mathcal{V}^{\bullet}(A)\right) \stackrel{\phi}{\leftarrow} Y_{\mathrm{Calc}_{\infty}^{0}}\left(C^{\bullet}(A)\right) \stackrel{\tau}{\leftarrow} Y_{\mathrm{Calc}_{\infty}^{0}}\left(\mathcal{V}^{\bullet}(A)\right) \stackrel{\psi}{\rightarrow} Y_{\mathrm{Calc}^{0}}\left(\mathcal{V}^{\bullet}(A)\right)
$$

where two-colored operads $\mathrm{Calc}_{\text {alg }}^{0}$, $\operatorname{Calc}_{\infty}^{0}$, Calc $^{0}$ are defined in 3.6. 
Next, we note that $C_{\bullet}(A)$ is an $A_{\infty}$ module over the $A_{\infty}$ algebra $C_{\bullet}\left(C^{\bullet}(A)\right)[[u]]$. We interpret this result as the existence of an $A_{\infty}$ morphism

$$
C_{\bullet}\left(C^{\bullet}(A)\right) \rightarrow Y_{\text {Calc }_{\text {alg }}^{0}}\left(\mathcal{V}^{\bullet}(A)\right)
$$

Then we observe that this is a quasi-isomorphism. The maps $\psi$ and $\tau$ in (3.1) are also quasi-isomorphisms; therefore we get an $A_{\infty}$ morphism from $Y\left(\mathcal{V}^{\bullet}(A)\right)$ to $C \bullet\left(C^{\bullet}(A)\right)$. To prove that this is a quasi-isomorphism, we are reduced to proving that $\phi$ is a quasi-isomorphism.

To that end, we study in some more detail the homology of the algebra $Y_{\text {Calco }_{\infty}^{0}}\left(\mathcal{A}^{\bullet}\right)$ for any Gerstenhaber algebra $\mathcal{A}^{\bullet}$ (Appendix). We show that this is a twisted version of the Hochschild homology, i.e. there is a spectral sequence starting with the latter and converging to the former. We observe that the map $\phi$ is filtered with respect to some filtration, and the fact that it is a quasi-isomorphism follows from considering the corresponding spectral sequence.

In the last subsection before Appendix, we modify the above arguments to prove the cyclic case of the main theorem.

3.1. Operads $G, G_{\infty}, G_{\text {alg }}, G_{\text {geom }}$. Here we recall the scheme of the proof of Theorem 2.4.1 which was used in $[\mathbf{T}]$, [T1].

Gerstenhaber algebras are algebras over an operad which we will denote by $G$.. In other words, $G(n)$ is the graded $k$-module of all $n$-ary operations composed of the product and the bracket in a Gerstenhaber algebra, subject to all relations following from Gerstenhaber algebra axioms. The operad $G$ is often denoted also by $e_{2}$.

By $G_{\infty}$ we denote the standard free resolution of $G$. This is an operad in the category of complexes. One description of it is as follows. Consider a graded space $\mathcal{A}^{\bullet}$. Let us pretend for a moment that $\mathcal{A}^{\bullet}$ is finite-dimensional. Consider the free graded Lie algebra $\operatorname{Lie}\left(\mathcal{A}^{\bullet}[1]^{*}\right)$ generated by the dual space to $\mathcal{A}^{\bullet}[1]$. Then the space $\mathcal{F}^{\bullet}\left(\mathcal{A}^{\bullet}\right)=\wedge^{\bullet} \operatorname{Lie}\left(\mathcal{A}^{\bullet}[1]^{*}\right)$ carries the structure of a Gerstenhaber algebra (Example 2.1.1). In fact $\mathcal{F}^{\bullet}\left(\mathcal{A}^{\bullet}\right)$ is the free Gerstenhaber algebra generated by $\mathcal{A}^{\bullet}[1]^{*}$. A $G_{\infty}$ structure on $\mathcal{A}^{\bullet}$ is by definition a derivation $\delta$ of the Gerstenhaber algebra $\mathcal{F}^{\bullet}\left(\mathcal{A}^{\bullet}\right)$ such that $|\delta|=1$ and $\delta^{2}=0$.

Remark 3.1.1. As stated, this definition has a problem if $\mathcal{A}^{\bullet}$ is infinitedimensional: it involves various linear maps from $\mathcal{A}^{\bullet}[1]^{*}$ to tensor powers of $\mathcal{A}^{\bullet}[1]^{*}$, satisfying certain relations. What we actually mean are dual maps from tensor powers of $\mathcal{A}^{\bullet}[1]$ to $\mathcal{A}^{\bullet}[1]$, satisfying dual relations. A rigorous definition can be given in the dual language of coalgebras. The same remark applies to the definitions and constructions of $3.6,3.10,4$ below.

Another, equivalent way of defining the operad $G_{\infty}$ is to put

$$
G_{\infty}=\operatorname{Cobar}\left(G^{\text {dual }}\right)
$$

where $G^{\text {dual }}$ is the Koszul dual operad as in $[\mathbf{G K}]$. Note that the operad $G$ is Koszul [GJ].

The operad $G_{\text {geom }}$ is the little discs operad [May]. To pass from it to an operad in the category of complexes, one defines the operad $C_{\bullet}\left(G_{\text {geom }}\right)$. It is known that the homology operad $H_{\bullet}\left(G_{\text {geom }}\right)$ is isomorphic to $G$, see $[\mathbf{A}],[\mathbf{C o}],[\mathbf{G J}]$.

The operad in the category of complexes $G_{\text {alg }}$ is the operad of all universal operations on the Hochschild cochain complex of an associative algebra. It has 
various versions each of which suits our purposes ([KS], [MS], [T1]). Let us start with the version from [MS]. Consider all multi-linear operations on Hochschild cochains which are linear combinations of iterated compositions of the following elementary operations:

$$
\begin{gathered}
o p(D)\left(a_{1}, \ldots, a_{d}\right)=a_{1} D\left(a_{2}, \ldots, a_{d}\right) \\
o p(D)\left(a_{1}, \ldots, a_{d}\right)=D\left(a_{1}, \ldots, a_{i} a_{i+1}, \ldots, a_{d}\right) \\
o p(D)\left(a_{1}, \ldots, a_{d}\right)=D\left(a_{1}, \ldots, a_{d-1}\right) a_{d} \\
o p(D, E)\left(a_{1}, \ldots, a_{d+e-1}\right)=D\left(a_{1}, \ldots, a_{i}, E\left(a_{i+1}, \ldots, a_{i+e}\right), \ldots\right) \\
o p(D, E)\left(a_{1}, \ldots, a_{d+e}\right)=D\left(a_{1}, \ldots, a_{d}\right) E\left(a_{d+1}, \ldots, a_{d+e}\right)
\end{gathered}
$$

(a minor technical point: this construction makes sense if one works with nonnormalized Hochschild cochains $\left.C^{\bullet}(A)=\operatorname{Hom}\left(A^{\otimes n}, A\right)\right)$. One can arrange these operations into an operad in the category of complexes, cf. [MS]. In [KS], a different version of this operad is proposed, namely the minimal operad $\mathcal{M}$. It consists of all universal operations on Hochschild cochains which are linear combinations of compositions of the brace operations from 2.5, the cup product, and the higher cup products which are defined if $A$ is an $A_{\infty}$ algebra. Such $n$-ary operations are naturally indexed by rooted trees whose vertices are labeled by symbols $1, \ldots, n, m_{i}$, $i \geq 2$, in such a way that each label from 1 to $n$ enters exactly once, and $m_{i}$ may only label vertices with $i$ outgoing edges. Finally, by $\mathcal{G}_{\text {alg }}$ we denote the standard free resolution of $G_{\text {alg }}$, the operad $\operatorname{Bar} \operatorname{Cobar}\left(G_{\text {alg }}\right)$. For any operad $\mathcal{O}$ in the category of complexes, the operad $\operatorname{Bar} \operatorname{Cobar}(\mathcal{O})$ admits an explicit description as in [KS] (cf. also 3.6).

The relation between the above operads is as follows.

$$
G_{\infty} \stackrel{f_{1}}{\rightarrow} G_{\text {alg }} \stackrel{g_{1}}{\longleftarrow} \mathcal{G}_{\text {alg }} \stackrel{g_{2}}{\rightarrow} C_{\bullet}\left(G_{\text {geom }}\right) \stackrel{F}{\rightarrow} G
$$

The quasi-isomorphism $g_{2}$ can be deduced from $[\mathbf{K S}]$, from $[\mathbf{M S}]$, or $[\mathbf{T 1}] ; g_{1}$ is the standard quasi-isomorphism between a resolution of an operad and the operad itself. $F$ is the formality quasi-isomorphism from $[\mathbf{K 1}],[\mathbf{T 1}]$; it depends on a choice of a Drinfeld associator. The existence of $f_{1}$ follow from the fact that , thanks to the existence of $F, G_{\infty}$ and $\mathcal{G}_{\text {alg }}$ are two free resolutions of $G$.

Therefore, since $G_{\text {alg }}$ acts on $C^{\bullet}(A)$, we see that $C^{\bullet}(A)$ is a $G_{\infty}$ algebra. This summarizes one of the versions of the proof of Theorem 2.4.1.

\subsection{Calculi.}

Definition 3.2.1. A precalculus is a pair of a Gerstenhaber algebra $\mathcal{V}^{\bullet}$ and a graded space $\Omega^{\bullet}$ together with

- a structure of a graded module over the graded commutative algebra $\mathcal{V}^{\bullet}$ on $\Omega^{-\bullet}$ (corresponding action is denoted by $i_{a}, a \in \mathcal{V}^{\bullet}$ );

- a structure of a graded module over the graded Lie algebra $\mathcal{V}^{\bullet+1}$ on $\Omega^{-\bullet}$ (corresponding action is denoted by $L_{a}, a \in \mathcal{V}^{\bullet}$ ) such that

$$
\left[L_{a}, i_{b}\right]=i_{[a, b]}
$$

and

$$
L_{a b}=L_{a} i_{b}+(-1)^{|a|} i_{a} L_{b}
$$


Definition 3.2.2. A calculus is a precalculus together with an operator $d$ of degree 1 on $\Omega^{\bullet}$ such that $d^{2}=0$ and

$$
\left[d, i_{a}\right]=L_{a} .
$$

ExAmple 3.2.3. For any manifold one defines a calculus $\operatorname{Calc}(M)$ with $\mathcal{V}^{\bullet}$ being the algebra of multivector fields, $\Omega^{\bullet}$ the space of differential forms, and $d$ the De Rham differential.

Example 3.2.4. For any associative algebra $A$ one defines a calculus $\operatorname{Calc}_{0}(A)$ by putting $\mathcal{V}^{\bullet}=H^{\bullet}(A, A)$ and $\Omega^{\bullet}=H_{\bullet}(A, A)$. The five operations from Definition 3.2.2 are the cup product, the Gerstenhaber bracket, the pairings $i_{D}$ and $L_{D}$, and the differential $B$, as in 3.3 below.

A differential graded $(\mathrm{dg})$ calculus is a calculus with extra differentials $\delta$ of degree 1 on $\mathcal{V}^{\bullet}$ and $b$ of degree -1 on $\Omega^{\bullet}$ which are derivations with respect to all the structures.

3.3. Pairings between chains and cochains. For a graded algebra $A$, for $D \in C^{d}(A, A)$, define

$$
i_{D}\left(a_{0} \otimes \ldots \otimes a_{n}\right)=(-1)^{|D| \sum_{i \leq d}\left(\left|a_{i}\right|+1\right)} a_{0} D\left(a_{1}, \ldots, a_{d}\right) \otimes a_{d+1} \otimes \ldots \otimes a_{n}
$$

Proposition 3.3.1.

$$
\begin{gathered}
{\left[b, i_{D}\right]=i_{\delta D}} \\
i_{D} i_{E}=(-1)^{|D||E|} i_{E \smile D}
\end{gathered}
$$

Now, put

$$
\begin{gathered}
L_{D}\left(a_{0} \otimes \ldots \otimes a_{n}\right)=\sum_{k=1}^{n-d} \epsilon_{k} a_{0} \otimes \ldots \otimes D\left(a_{k+1}, \ldots, a_{k+d}\right) \otimes \ldots \otimes a_{n}+ \\
\sum_{k=n+1-d}^{n} \eta_{k} D\left(a_{k+1}, \ldots, a_{n}, a_{0}, \ldots\right) \otimes \ldots \otimes a_{k}
\end{gathered}
$$

(The second sum in the above formula is taken over all cyclic permutations such that $a_{0}$ is inside $D$ ). The signs are given by

$$
\epsilon_{k}=(|D|+1) \sum_{i=0}^{k}\left(\left|a_{i}\right|+1\right)
$$

and

$$
\eta_{k}=|D|+1+\sum_{i \leq k}\left(\left|a_{i}\right|+1\right) \sum_{i \geq k}\left(\left|a_{i}\right|+1\right)
$$

Proposition 3.3.2.

$$
\begin{gathered}
{\left[L_{D}, L_{E}\right]=L_{[D, E]}} \\
{\left[b, L_{D}\right]+L_{\delta D}=0} \\
{\left[L_{D}, B\right]=0}
\end{gathered}
$$


Now let us extend the above operations to the cyclic complex. Define

$$
\begin{gathered}
S_{D}\left(a_{0} \otimes \ldots \otimes a_{n}\right)=\sum_{j \geq 0 ; k \geq j+d} \epsilon_{j k} 1 \otimes a_{k+1} \otimes \ldots a_{0} \otimes \ldots \otimes \\
\otimes D\left(a_{j+1}, \ldots, a_{j+d}\right) \otimes \ldots \otimes a_{k}
\end{gathered}
$$

(The sum is taken over all cyclic permutations for which $a_{0}$ appears to the left of $D)$. The signs are as follows:

$\epsilon_{j k}=|D|\left(\left|a_{0}\right|+\sum_{i=1}^{n}\left(\left|a_{i}\right|+1\right)\right)+(|D|+1) \sum_{j+1}^{k}\left(\left|a_{i}\right|+1\right)+\sum_{i \leq k}\left(\left|a_{i}\right|+1\right) \sum_{i \geq k}\left(\left|a_{i}\right|+1\right)$

As we will see later, all the above operations are partial cases of a unified algebraic structure for chains and cochains, cf. 3.8; the sign rule for this unified construction was explained in 2.6.

Proposition 3.3.3. ([R])

$$
\left[b+u B, i_{D}+u S_{D}\right]-i_{\delta D}-u S_{\delta D}=L_{D}
$$

Proposition 3.3.4. ([DGT]) There exists a linear transformation $T(D, E)$ of the Hochschild chain complex, bilinear in $D, E \in C^{\bullet}(A, A)$, such that

$$
\begin{gathered}
{[b+u B, T(D, E)]-T(\delta D, E)-(-1)^{|D|} T(D, \delta E)=} \\
\quad=\left[L_{D}, i_{E}+u S_{E}\right]-(-1)^{|D|+1}\left(i_{[D, E]}+u S_{[D, E]}\right)
\end{gathered}
$$

\subsection{The calculus $\operatorname{Calc}(A)$.}

Theorem 3.4.1. [TTT] For any associative algebra $A$ one can define a dg calculus $\operatorname{Calc}(A)$ such that:

1). As dg Lie algebras, $\mathcal{V}^{\bullet+1}(A)$ is quasi-isomorphic to the Hochschild cochain complex $C^{\bullet+1}(A)$. As dg modules over the dg Lie algebra $\mathcal{V}^{\bullet+1}(A), \Omega^{\bullet}(A)[[u]]$ with the differential $\delta+u B$ is quasi-isomorphic to the negative cyclic complex $C \cdot(A)[[u]]$ with the differential $b+u B$.

2). If $A=C^{\infty}(M)$ then there is a quasi-isomorphism of calculi

$$
\operatorname{Calc}(A) \rightarrow \operatorname{Calc}(M)
$$

3). For any $A$, the calculus $H^{\bullet}(\operatorname{Calc}(A))$ is isomorphic to the calculus $\operatorname{Calc}_{0}(A)$ from Example 3.2.4.

3.5. Two-colored operads. The notion of a two-colored operad formalizes the situation when one has an algebra $A$ over an operad $\mathcal{O}$, an object $B$, and a set $\mathcal{M}(n)$ of operations $A^{\otimes n} \otimes B \rightarrow B$. The union of $\mathcal{M}(n)$ is supposed to be closed under some natural operations.

More precisely, a two-colored operad $(\mathcal{O}, \mathcal{M})$ consists of an operad $\mathcal{O}$ and a collection of objects $\mathcal{M}(n), n \geq 0$, together with an action of the symmetric group $S_{n}$ on $\mathcal{M}(n)$ for all $n$, and with the operations

$$
\begin{gathered}
\mathcal{M}(k) \otimes \mathcal{O}\left(n_{1}\right) \otimes \ldots \otimes \mathcal{O}\left(n_{k}\right) \rightarrow \mathcal{M}\left(n_{1}+\ldots+n_{k}\right) \\
\mathcal{M}(k) \otimes \mathcal{M}(l) \rightarrow \mathcal{M}(k+l)
\end{gathered}
$$

subject to natural conditions of associativity and symmetry with respect to permutations. 
An algebra over a two-colored operad $(\mathcal{O}, \mathcal{M})$ is a pair of objects $(A, B)$ such that $A$ is an $\mathcal{O}$-algebra, together with operations

$$
\mathcal{M}(n) \otimes A^{\otimes n} \otimes B \rightarrow B
$$

subject to natural relations.

One can easily adapt the basic notions of the theory of operads to this context. For example, the free two-colored operad generated by a collection of $k\left[S_{n}\right]$-modules $\mathcal{Q}(n), \mathcal{R}(n)$ is the pair $(\mathcal{O}, \mathcal{M})$ where $\mathcal{O}$ is the free operad generated by $\{\mathcal{Q}(n)\}$ and $\mathcal{M}(n)$ is described as follows. Consider all rooted trees with a chosen path $\mathbf{p}_{0}$ from the root to an external vertex $\mathbf{v}_{0}$. Let the external vertices other than $\mathbf{v}_{0}$ be numbered by $1, \ldots, n$. We will call such objects two-colored trees. For such a tree, put

where

$$
\mathcal{M}(T)=\bigotimes_{\text {internal vertices } v} \mathcal{M}(v),
$$

$$
\mathcal{M}(v)=\mathcal{Q}(\#(\text { edges outgoing from } v))
$$

if $v$ is not on $\mathbf{p}_{0}$;

$$
\mathcal{M}(v)=\mathcal{R}(\#(\text { edges outgoing from } v))
$$

if $v$ is on $\mathbf{p}_{0} \cdot \mathcal{M}(n)$ is the direct sum of $\mathcal{M}(T)$ over all isomorphism classes of such trees.

If $(\mathcal{O}, \mathcal{M})$ is a two-colored dg operad, then the cofree two-colored cooperad cogenerated by $(\mathcal{O}, \mathcal{M})$ acquires a differential. This differential is the sum of the one induced from $(\mathcal{O}, \mathcal{M})$ and the new one, which sends an element of the direct summand corresponding to a (two-colored) tree $T$ to the sum of elements in direct summands corresponding to trees $T^{\prime}$ obtained from $T$ by contracting an internal edge. These elements are obtained from the original element by applying an appropriate composition in the operad $(\mathcal{O}, \mathcal{M})$. Thus one gets a two-colored cooperad in the category of complexes $\operatorname{Cobar}(\mathcal{O}, \mathcal{M})$.

Dually, if $(\mathcal{O}, \mathcal{M})$ is a two-colored dg cooperad, one constructs a two-colored dg operad $\operatorname{Bar}(\mathcal{O}, \mathcal{M})$. Its underlying space is a direct sum over (two-colored) trees, and the new component of the differential consists of inserting an internal edge in all possible positions, combined with an appropriate cooperadic cocomposition.

Composing these two constructions, one produces for a dg two-colored operad $(\mathcal{O}, \mathcal{M})$ a new dg two-colored operad $\operatorname{Bar} \operatorname{Cobar}(\mathcal{O}, \mathcal{M})$. This is the standard free resolution of $(\mathcal{O}, \mathcal{M})$, which means that it is free as an operad in the category of graded vector spaces and that there is a canonical quasi-isomorphism of operads

$$
\operatorname{Bar} \operatorname{Cobar}(\mathcal{O}, \mathcal{M}) \rightarrow(\mathcal{O}, \mathcal{M}) .
$$

Explicitly (compare [KS]), this resolution is the direct sum of components numbered by (two-colored) trees whose edges are labeled by one of the two labels, finite or infinite. All external edges are infinite. The terms in the new differential are of two types: a) contracting a finite edge, combined with an operadic composition; b) making a finite edge infinite.

One can also define, following [GK], a Koszul dual cooperad of a two-colored operad, and extend the notion of a Koszul operad into the two-colored setting. For a Koszul two-colored operad $(\mathcal{O}, \mathcal{M})$, the $\mathrm{dg}$ operad $\operatorname{Bar}\left((\mathcal{O}, \mathcal{M})^{\text {dual }}\right)$ is a free resolution of $(\mathcal{O}, \mathcal{M})$. 
3.6. Two-colored operads Calc, $\mathrm{Calc}_{\infty}, \mathrm{Calc}_{\mathrm{geom}}, \mathrm{Calc}_{\mathrm{alg}}$. In this section we will extend the method that was outlined in 3.1. This will enable us to prove both Theorem 3.4.1, 1)-3), and Theorem 2.7.1. To prove statement 4) of Theorem 3.4.1, some additional work is needed; we will give a proof in a subsequent paper.

By Calc, resp. Calc ${ }^{0}$, we denote the two-colored operad in the category of graded spaces such that algebras over them are calculi (resp. precalculi). In other words, $\mathcal{O}=G$ and $\mathcal{M}(n)$ consists of all $n$-ary operations composed of $i_{a}, L_{a}$, and $d\left(\right.$ resp. $i_{a}$ and $\left.L_{a}\right)$.

By $\mathrm{Calc}_{\infty}$ we denote the standard resolution of Calc. One can write it as

$$
\text { Calc }_{\infty}=\text { Bar Calc }^{\text {dual }}
$$

Similarly,

$$
\mathrm{Calc}_{\infty}^{0}=\text { Bar Calc }^{0^{\text {dual }}}
$$

(one can show that Calc and $\mathrm{Calc}^{0}$ are Koszul).

Alternatively, one can give the following explicit definition.

A Calc $\infty$ algebra is a pair of graded vector spaces $\left(\mathcal{A}^{\bullet}, \mathcal{B}^{\bullet}\right)$ where $\mathcal{A}^{\bullet}$ is a $G_{\infty}$ algebra, together with the following extra data. As in 3.1, let us pretend that $\mathcal{A}^{\bullet}, \mathcal{B}^{\bullet}$ are finite-dimensional (cf. Remark 3.1.1). Recall from 3.1 that one can define the $\mathrm{dg}$ Gerstenhaber algebra $\mathcal{F}^{\bullet}\left(\mathcal{A}^{\bullet}\right)$. Let $\Omega_{\mathcal{A}^{\bullet}, \mathcal{B}^{\bullet}}^{-\bullet}$ be the free graded $Y\left(\mathcal{F}^{\bullet}\left(\mathcal{A}^{\bullet}\right)\right)$-module generated by $\left(\mathcal{B}^{-\bullet}[1]\right)^{*}$. In other words, let $\left(\mathcal{F}^{\bullet}\left(\mathcal{A}^{\bullet}\right), \Omega_{\mathcal{A}}^{\bullet}, \mathcal{B}^{\bullet}\right)$ be the free precalculus generated by $\left(\mathcal{A}^{-\bullet}[1]\right)^{*}$ and $\left(\mathcal{B}^{-\bullet}[1]\right)^{*}$.

A $\operatorname{Calc}_{\infty}^{0}$ algebra structure on $\left(\mathcal{A}^{\bullet}, \mathcal{B}^{\bullet}\right)$ is a linear operator $d$ on $\Omega_{\mathcal{A}}^{\bullet}, \mathcal{B}_{\bullet}$ of square zero and degree one, such that $\left(\Omega_{\mathcal{A} \bullet, \mathcal{B}^{\bullet}}^{-\bullet}, d\right)$ is a dg module over the $\mathrm{dg}$ algebra $\left(Y\left(\mathcal{F}^{\bullet}\left(\mathcal{A}^{\bullet}\right)\right), \delta\right)$.

Let $u$ be a formal parameter of degree two. A Calc $\infty$ algebra structure on $\left(\mathcal{A}^{\bullet}, \mathcal{B}^{\bullet}\right)$ is a $k[[u]]$-linear, $(u)$-adically continuous operator $d$ on $\Omega_{\mathcal{A} \bullet, \mathcal{B} \bullet}^{\bullet}[[u]]$ of square zero and degree one, such that $\left(\Omega_{\mathcal{A}}^{-\bullet} \boldsymbol{B}_{\bullet} \cdot[[u]], d\right)$ is a dg module over the dg algebra $\left(Y\left(\mathcal{F}^{\bullet}\left(\mathcal{A}^{\bullet}\right)\right)[[u]], \delta+u d\right)$.

We define $\operatorname{Calc}_{\text {geom }}^{0}=(\mathcal{O}, \mathcal{M})$ where $\mathcal{O}$ is the little discs operad and $\mathcal{M}$ is the configuration space, up to dilations, of cylinders $[0, r] \times S^{1}$ with $n$ disjoint discs in the interior. The operations of type (3.6) consist of inserting little discs into the discs on the cylinder. The operations of type (3.7) consist of putting one cylinder on top of the other.

As for Calc geom, $\mathcal{O}$ is the little discs operad and $\mathcal{M}$ is the configuration space, up to dilations and horizontal rotations, of cylinders $[0, r] \times S^{1}$ with a marked point on each component of the boundary and with $n$ disjoint discs in the interior. The operations of type (3.6) consist of inserting little discs into the discs on the cylinder. The operations of type (3.7) consist of aligning marked points and then putting one cylinder on top of the other.

Now let us indicate how one defines Calc $\mathrm{c}_{\mathrm{alg}}^{0}$ and Calcalg. For them, $\mathcal{O}=G_{\mathrm{alg}}$. To describe $\mathcal{M}$, consider all universal operations $C^{\bullet}(A)^{\otimes n} \otimes C_{\bullet}(A) \rightarrow C_{\bullet}(A)$ which are linear combinations of iterated compositions of the operations from $G_{\text {alg }}$ with the following elementary operations:

$$
\begin{gathered}
a_{0} \otimes \ldots \otimes a_{p} \mapsto 1 \otimes a_{0} \otimes \ldots \otimes a_{p} \\
a_{0} \otimes \ldots \otimes a_{p} \mapsto a_{p} \otimes a_{0} \otimes \ldots \otimes a_{p-1} \\
a_{0} \otimes \ldots \otimes a_{p} \mapsto a_{0} a_{1} \otimes a_{2} \otimes \ldots \otimes a_{p}
\end{gathered}
$$




$$
\left(D, a_{0} \otimes \ldots \otimes a_{p}\right) \mapsto a_{0} \otimes D\left(a_{1}, \ldots, a_{d}\right) \otimes a_{d+1} \otimes \ldots \otimes a_{p}
$$

(As in 3.1, to make it correct, one has to work with non-normalized chains and cochains).

For the two-colored operad Calc alg, the $\mathcal{M}$ part consists only of the operations for which the term containing $a_{0}$ remains on the position number zero.

For example, the operation

$$
\left(D_{1}, D_{2}, D_{3}, a_{0} \otimes \ldots \otimes a_{7}\right) \mapsto D_{1}\left(a_{7}, a_{0}\right) D_{3}\left(a_{1}, D_{2}\left(a_{2}, a_{3}\right)\right) a_{4} \otimes a_{5} a_{6}
$$

is in $\mathcal{M}$ for both Calc alg and $\mathrm{Calc}_{\mathrm{alg}}^{0}$; the operation

$$
\left.\left(D_{1}, D_{2}, a_{0} \otimes \ldots \otimes a_{5}\right) \mapsto a_{1} a_{2} \otimes a_{3} \otimes D_{2}\left(a_{4}, D_{1}\left(a_{5}\right), a_{0}\right)\right)
$$

is in $\mathcal{M}$ for Calcalg but not for Calc $_{\text {alg }}^{0}$ (the term containing $a_{0}$ is on the position number two); the operation

$$
\left(D_{1}, D_{2}, D_{3}, a_{0} \otimes \ldots \otimes a_{5}\right) \mapsto D_{1}\left(a_{5} a_{0}\right) a_{1} \otimes a_{2} D_{3}\left(a_{4}, D_{2}\left(a_{3}\right)\right)
$$

is not in $\mathcal{M}$ for either (because the cyclic order of $a_{i}$ 's is broken).

As in 3.1, a minimal version in the manner of $[\mathbf{K S}]$ can be defined (and is necessary for the current version of the proof of the main theorem). We will discuss it in full in a more detailed exposition.

Finally, put

$$
\mathcal{C}_{\text {alg }}=\text { Bar Cobar }\left(\text { Calc }_{\text {alg }}\right)
$$

Adapting the arguments from $[\mathbf{M S}]$, $[\mathbf{T} \mathbf{1}]$ or from $[\mathbf{K S}]$ to our purposes, one proves that the following chain of quasi-isomorphisms extends that of 3.1:

$$
\text { Calc }_{\infty} \stackrel{f_{1}}{\rightarrow} \text { Calc }_{\text {alg }} \stackrel{g_{1}}{\leftarrow} \mathcal{C}_{\text {alg }} \stackrel{g_{2}}{\rightarrow} C_{\bullet}\left(\text { Calc }_{\text {geom }}\right) \stackrel{F}{\rightarrow} \text { Calc }
$$

and similarly for Calc ${ }^{0}$. As a corollary, one gets Theorem 3.4.1.

3.7. Enveloping algebra of an algebra over a two-colored operad. Let $\mathcal{P}=(\mathcal{O}, \mathcal{M})$ be a two-colored dg operad. For an $\mathcal{O}$-algebra $\mathcal{A}^{\bullet}$ put

$$
Y_{\mathcal{P}}\left(\mathcal{A}^{\bullet}\right)=\oplus_{n>0} \mathcal{A}^{\bullet \otimes n} \otimes_{k\left[S_{n}\right]} \mathcal{M}(n) / \sim
$$

where the equivalence relation $\sim$ is generated by the following:

for $a_{1}, \ldots, a_{n} \in \mathcal{A}^{\bullet}, m \in \mathcal{M}(k), o \in \mathcal{O}(l), k+l-1=n$, and for all $i$

$$
\left(a_{1} \otimes \ldots \otimes a_{n}\right) \otimes\left(o \circ_{i} m\right) \sim\left(a_{1} \otimes \ldots \otimes a_{i-1} \otimes o\left(a_{i}, \ldots, a_{i+l-1}\right) \otimes \ldots \otimes a_{n}\right) \otimes m
$$

taken with the appropriate sign, where $o_{i}: \mathcal{O}(l) \otimes \mathcal{M}(k) \rightarrow \mathcal{M}(n)$ is the $i$ th elementary composition. algebra.

The operations $\mathcal{M}(k) \otimes \mathcal{M}(l) \rightarrow \mathcal{M}(k+l)$ turn $Y_{\mathcal{P}}\left(\mathcal{A}^{\bullet}\right)$ into an associative

EXAmPle 3.7.1. . Let $\mathcal{P}=(\mathrm{As}, \mathcal{M})$ be the two-colored operad algebras over which are pairs $(A, B)$, where $A$ is an associative algebra and $B$ is a left $A$-module (resp. a right module, resp. a bimodule). Then $Y_{\mathcal{P}}(A)=A$ (resp. $A^{\text {op }}$, resp. $\left.A \otimes A^{\mathrm{op}}\right)$.

EXAMPLE 3.7.2. . Let $\mathcal{P}=($ Lie, $\mathcal{M})$ be the two-colored operad algebras over which are pairs $(\mathfrak{g}, B)$ where $\mathfrak{g}$ is a Lie algebra and $B$ is a $\mathfrak{g}$-module. Then $Y_{\mathcal{P}}(\mathfrak{g})=$ $U(\mathfrak{g})$.

EXAmple 3.7.3. Let $\mathcal{P}=\operatorname{Calc}^{0}$. Then, for a Gerstenhaber algebra $\mathcal{A}^{\bullet}, Y_{\mathcal{P}}\left(\mathcal{A}^{\bullet}\right)=$ $Y\left(\mathcal{A}^{\bullet}\right)$ 
Example 3.7.4. Let $\mathcal{P}=$ Calc. Denote by $\mathfrak{a}$ a one-dimensional Abelian graded Lie algebra concentrated in degree one. This algebra acts on $Y\left(\mathcal{A}^{\bullet}\right)$ by derivations, the generator acting by $d$. One can form a cross product $U(\mathfrak{a}) \ltimes Y\left(\mathcal{A}^{\bullet}\right)$. For a Gerstenhaber algebra $\mathcal{A}^{\bullet}$,

$$
Y_{\mathcal{P}}\left(\mathcal{A}^{\bullet}\right) \simeq U(\mathfrak{a}) \ltimes Y\left(\mathcal{A}^{\bullet}\right) .
$$

3.8. The $A_{\infty}$ module structure on Hochschild chains. Recall the definition of $A_{\infty}$ modules over $A_{\infty}$ algebras. First, note that for a graded space $\mathcal{M}$, the Gerstenhaber bracket [, ] can be extended to the space

$$
\operatorname{Hom}\left(\overline{\mathcal{C}}^{\otimes \bullet}, \mathcal{C}\right) \oplus \operatorname{Hom}\left(\mathcal{M} \otimes \overline{\mathcal{C}}^{\otimes \bullet}, \mathcal{M}\right)
$$

For a graded $k$-module $\mathcal{M}$, a structure of an $A_{\infty}$ module over an $A_{\infty}$ algebra $\mathcal{C}$ on $\mathcal{M}$ is a cochain

$$
\begin{gathered}
\mu=\sum_{n=1}^{\infty} \mu_{n} \\
\mu_{n} \in \operatorname{Hom}\left(\mathcal{M} \otimes \overline{\mathcal{C}}^{\otimes n-1}, \mathcal{M}\right)
\end{gathered}
$$

such that

$$
[m+\mu, m+\mu]=0
$$

Theorem 3.8.1. On $C \cdot(A)[[u]]$, there exists a structure of an $A_{\infty}$ module over the $A_{\infty}$ algebra $C_{\bullet}\left(C^{\bullet}(A)\right)[[u]]$ such that:

- All $\mu_{n}$ are $k[[u]]$-linear, $(u)$-adically continuous

- $\mu_{1}=b+u B$ on $C \cdot(A)[[u]]$

$$
\text { For } a \in C_{\bullet}(A)[[u]] \text { : }
$$

- $\mu_{2}(a, D)=(-1)^{|a||D|+|a|}\left(i_{D}+u S_{D}\right) a$

- $\mu_{2}(a, 1 \otimes D)=(-1)^{|a||D|} L_{D} a$

For $a, x \in C_{\bullet}(A)[[u]]$ :

$$
(-1)^{|a|} \mu_{2}(a, x)=\left(\operatorname{sh}+u \operatorname{sh}^{\prime}\right)(a, x)
$$

To obtain formulas for the structure of an $A_{\infty}$ module from Theorem 3.8.1, one has to assume that, in the formulas for the $A_{\infty}$ structure from Theorem 2.6.1, all $D_{j}^{(1)}$ are elements of $A$; then one has to replace braces \{\} by the usual parentheses ( ) symbolizing evaluation of a multi-linear map at elements of $A$.

3.9. The algebra $Y_{\text {Calc }_{\text {alg }}^{0}}\left(C^{\bullet}(A)\right)$ and the Hochschild complex. Note that the construction from 3.8 can be interpreted as existence of an $A_{\infty}$ morphism

$$
C_{\bullet}\left(C^{\bullet}(A)\right) \rightarrow Y_{\text {Calc }_{\text {alg }}^{0}}\left(C^{\bullet}(A)\right)
$$

From (3.9) one gets an algebra homomorphism

$$
Y_{\mathrm{Calc}_{\infty}^{0}}\left(C^{\bullet}(A)\right) \rightarrow Y_{\mathrm{Calc}_{\mathrm{alg}}^{0}}\left(C^{\bullet}(A)\right)
$$

It is easy to show that both maps

$$
Y_{\operatorname{Calc}_{\infty}^{0}}\left(C^{\bullet}(A)\right) \leftarrow Y_{\text {Calc }_{\infty}^{0}}\left(\mathcal{V}^{\bullet}(A)\right) \rightarrow Y_{\mathrm{Calc}^{0}}\left(\mathcal{V}^{\bullet}(A)\right)
$$

are quasi-isomorphisms. To prove the first part of Theorem 2.7.1, it remains to show that (3.11) and (3.10) are quasi-isomorphisms under our assumptions. 
3.10. The algebra $Y_{\text {Calc }_{\infty}^{0}}\left(C^{\bullet}(A)\right)$ and the Hochschild complex. To show that (3.11) is a quasi-isomorphism, we introduce the following filtrations: on $C_{\bullet}\left(C^{\bullet}(A)\right)$, let

$$
\text { filt }_{n}=C_{\leq n}\left(C^{\bullet}(A)\right)=C^{\bullet}(A) \otimes{\overline{C^{\bullet}(A)}}^{\otimes \leq n}
$$

On $Y_{\mathrm{Calc}_{\mathrm{alg}}^{0}}\left(C^{\bullet}(A)\right)$ and $Y_{\mathrm{Calc}_{\infty}^{0}}\left(C^{\bullet}(A)\right)$, let

$$
\text { filt }_{n}=\sum_{m \leq n} \mathcal{Q}(m) \otimes_{k\left[S_{m}\right]} C^{\bullet}(A)^{\otimes m} / \sim
$$

It is easy to see that (3.10) preserves the filtration and therefore induces an isomorphism of associated graded quotients. The morphism (3.11) preserves the filtration by definition. At the level of associated graded quotients, (3.11) induces a morphism of complexes

$$
Y_{\mathrm{Calc}_{\infty}^{0}}\left(H^{\bullet}(A)\right) \rightarrow C_{\bullet}\left(H^{\bullet}(A)\right)
$$

where, in the left hand side, $H^{\bullet}(A)$ is the Hochschild cohomology, viewed as a Gerstenhaber algebra on which all the operations are zero. The fact that (3.15) is an isomorphism follows from Proposition 4.2.4 in the Appendix.

3.11. Another proof of quasi-isomorphicity of the map (3.11). We are going to prove a slightly more general statement.

Proposition 3.11.1. Let $U$ be an arbitrary $G_{\mathrm{alg}}$-algebra. Then the map $Y_{\mathrm{Calc}_{\infty}^{0}}(U) \rightarrow Y_{\mathrm{Calc}_{\mathrm{alg}}^{0}}(U)$ constructed in the same way as the map (3.11) is a quasiisomorphism.

This proof is based on two facts. The first one is that the map of colored operads $f_{1}$ from (3.9) is a quasi-isomorphism. The second fact says that $M_{\mathrm{alg}}^{0}$ is free over $G_{\text {alg }}$. This means that one can choose $S_{n}$-equivariant subspaces $E(n) \subset M_{\text {alg }}^{0}(n)$ (for any $n$ ) in such a way that for all $N$ the insertion map

$$
\bigoplus_{n, M_{1}+M_{2}+\cdots+M_{n}=N} M_{\text {alg }}^{0}(n) \otimes_{S_{n}} G_{\text {alg }}\left(M_{1}\right) \otimes \cdots \otimes G_{\text {alg }}\left(M_{n}\right) \otimes_{S_{M_{1}} \times \cdots \times S_{M_{n}}} k\left[S_{N}\right] \rightarrow M_{\text {alg }}^{0}(N)
$$

is an isomorphism. This fact follows from the explicit construction of Calcalg.

Having these two facts, we prove the Proposition as follows. First, let us translate the definition of a universal enveloping algebra into the language of PROPs. Let $(\mathcal{O}, M)$ be a two-colored operad and $U$ be an $\mathcal{O}$-algebra. Let $P_{\mathcal{O}}$ be the PROP generated by $\mathcal{O}$. Then the structure maps

$$
\mathcal{O}\left(n_{1}\right) \otimes \cdots \otimes \mathcal{O}\left(n_{m}\right) \otimes M(m) \rightarrow M\left(n_{1}+\cdots+n_{m}\right)
$$

endow $M$ with a structure of a functor $M^{\prime}: P_{\mathcal{O}}^{\text {op }} \rightarrow$ Complexes, where for $[n] \in$ $\mathrm{Ob} P_{\mathcal{O}}, M^{\prime}([n])=M(n)$. Further on, we will denote $M^{\prime}$ by $M$. Put $F_{U}([n])=U^{\otimes n}$. Then, since $U$ is a $P_{\mathcal{O}}$-algebra, $F_{U}$ is a functor $P_{\mathcal{O}} \rightarrow$ Complexes.

We see that

$$
Y_{\mathcal{O}, M}(U) \cong F_{U} \otimes_{P_{\mathcal{O}}} M,
$$

where on the right hand side we use the MacLane tensor product.

We have a quasi-isomorpism $f_{1}: \mathrm{Calc}_{\infty}^{0} \rightarrow \mathrm{Calc}_{\text {alg }}^{0}$. It produces a symmetric monoidal functor $F: P_{G_{\infty}} \rightarrow P_{G_{\text {alg }}}$ which induces a quasi-isomorphism on the spaces of homomorphisms; also, $f_{1}$ gives rise to a natural transformation $G: M_{\infty}^{0} \rightarrow$ $M_{\text {alg }}^{0} \circ F$ which is a quasi-isomorphism. 
Let us come back to our $G_{\text {alg-algebra }} U$. Let $U^{\prime}$ be $U$ considered as a $G_{\infty^{-}}$ algebra, where the corresponding structure is induced by $f_{1}$. We have:

$$
F_{U^{\prime}} \cong F_{U} \circ F \text {. }
$$

In this light, the map $Y_{\mathrm{Calc}_{\infty}^{0}}\left(U^{\prime}\right) \rightarrow Y_{\mathrm{Calc}_{\text {alg }}^{0}}(U)$, which coincides with the map (3.11), is described as the composition:

$$
M_{\infty}^{0} \otimes_{P_{G_{\infty}}} F_{U^{\prime}} \rightarrow M_{\text {alg }}^{0} \circ F \otimes_{P_{G_{\infty}}} F_{U} \circ F \rightarrow M_{\text {alg }}^{0} \otimes_{P_{G_{\text {alg }}}} F_{U},
$$

where the first map is induced by $G$ and (3.16).

We need to show that this composition produces a quasi-isomorphism. To this end, we introduce a functor $F_{\text {! }}$ from the category of functors $P_{G_{\infty}} \rightarrow$ Complexes to the category of functors $P_{G_{\text {alg }}} \rightarrow$ Complexes. Denote $h_{[m]}([n])=\operatorname{hom}_{P_{G_{\text {alg }}}}([n],[m])$. Each $h_{[m]}$ is a functor $P_{G_{\text {alg }}}^{\text {op }} \rightarrow$ Complexes. For a functor $N: P_{G_{\infty}} \rightarrow$ Complexes set $F_{!} N([m]):=N \otimes_{P_{G_{\infty}}} h_{[m]}$. We have canonical isomorphisms

$$
\operatorname{hom}_{P_{G_{\text {alg }}}}(F ! N, M) \cong \operatorname{hom}_{P_{G_{\infty}}}(N, M \circ F)
$$

for any $M: P_{G_{\text {alg }}} \rightarrow$ Complexes; and

$$
F_{!} M \otimes_{P_{G_{\text {alg }}}} L \cong M \otimes_{P_{G_{\infty}}}(L \circ F) .
$$

In particular, the map $G$ induces a map $G^{\prime}: F_{!} M_{\infty}^{0} \rightarrow M_{\text {alg }}^{0}$. Since $F$ is a quasiisomorphism and $M_{\infty}^{0}$ is semi-free and has a set of generators centered in nonpositive degrees, it follows that $G^{\prime}$ is a quasi-isomorphism. One sees that the map in (3.17) can be rewritten as the following composition:

$$
M_{\infty}^{0} \otimes_{P_{G_{\infty}}} F_{U^{\prime}} \cong M_{\infty}^{0} \otimes_{P_{G_{\infty}}} F_{U} \circ F \cong F_{!} M_{\infty}^{0} \otimes_{P_{G_{\text {alg }}}} F_{U} \rightarrow M_{\mathrm{alg}}^{0} \otimes_{P_{G_{\mathrm{alg}}}} F_{U} .
$$

where the second map is induced by $G^{\prime}$. Since $G^{\prime}$ is a quasi-isomorphism and $M_{\mathrm{alg}}^{0}$ is semi-free and has a set of generators centered in non-positive degrees, the second map is a quasi-isomorphism, therefore, the whole composition is a quasiisomorphism.

3.12. (3.10) is a quasi-isomorphism. Again, we we will replace $C^{\bullet}(A, A)$ with an arbitrary $G_{\text {alg-algebra }} U$. $U$ has an associative cup-product, and we can form the Hochschild chain complex $C_{\bullet}(U)$ with respect to this product. The map (3.10) is then generalized to a map $\phi:=\phi_{U}: C_{\bullet}(U) \rightarrow Y_{\text {Calc }^{0}}(U)$, and we need to show that this map is a quasi-isomorphism. We are going to make a couple of reductions. Firstly, we can use the filtration as in (3.13) by the number of tensor factors of $U$. Then it suffices to check that $\phi$ induces a quasi-isomorphism of the associated graded spaces. This implies that it suffices to consider the case in which all operations on $U$ vanish. To prove this statement we need one more reduction. Note that any commutative algebra $B$ can be considered as a $G_{\text {alg-algebra in which }}$ the cup product is commutative and all braces vanish. Denote thus obtained $G_{\text {alg- }}$ algebra by $B^{\prime}$. We have $U=B^{\prime}$, where $B$ has zero product. We now want to reduce this case to the case in which $U=(S V)^{\prime}$, where $S V$ is a free commutative algebra. To this end we notice that for $B$ having zero product, there exists a semifree resolution $p: S W \rightarrow B$ having the property that $S W$ can be endowed with an increasing exhausting filtration $F$ such that the associated graded algebra $\mathrm{Gr} S W$ is free. Since the functors $C$. and $Y_{\mathrm{Calc}^{0}}$ preserve quasi-isomorphisms, it suffices to show that $\phi_{S W}$ is a quasi-isomorphism; using the filtration induced by $F$, we reduce the statement to quasi-isomorphicity of $\phi_{\mathrm{Gr} S W}$, where $\operatorname{Gr} S W$ is free. 
Thus, from now on we set $U=(S V)^{\prime}$. In this case we have the KostantHochschild-Rosenberg quasi-isomorphism $\omega: S V \otimes S(V[1]) \rightarrow C_{\bullet}(S V)$. Compute the composition $\omega \phi: S V \otimes S(V[1]) \rightarrow Y_{\mathrm{Calc}^{0}}(S V)$. To this end, we are going to use the elements $i, L \in M_{\mathrm{alg}}^{0}(1)$ defined in (3.2.1). Then it is easy to check that $\omega \phi$ is homotopy equivalent to the map $\psi$, which on an element $u \otimes\left(a_{1} \wedge \cdots \wedge a_{n}\right)$, where $u \in S V$ and $a_{i} \in V$, takes the value

$$
i_{u} \operatorname{Alt}\left(L_{a_{i_{1}}} L_{a_{i_{2}}} \ldots L_{a_{i_{n}}}\right),
$$

where Alt means alternation.

Let us now use the quasi-isomorphisms $f_{1}:$ Calc $_{\infty}^{0} \rightarrow \operatorname{Calc}_{a l g}^{0}$ from (3.10) and $f: Y_{\mathrm{Calc}_{\infty}^{0}}(S V) \rightarrow Y_{\mathrm{Calc}_{\mathrm{alg}}^{0}}(S V)$ as in (3.11). Let $I^{\prime} \in M_{\infty}^{0}(1), L^{\prime} \in M_{\infty}^{0}(1)$ be such that $f_{1}\left(I^{\prime}\right)$ (resp. $f_{1}\left(L^{\prime}\right)$ ) is homologous in $M_{\text {alg }}^{0}(1)$ to $i$ (resp. $L$ ).

Define a map $\chi: S V \otimes S(V[1]) \rightarrow Y_{\mathrm{Calc}_{\infty}^{0}}(S V)$ by setting

$$
\chi\left(u \otimes\left(a_{1} \wedge \cdots a_{n}\right)\right)=I_{u}^{\prime} \operatorname{Alt}\left(L_{a_{i_{1}}}^{\prime} L_{a_{i_{2}}}^{\prime} \ldots L_{a_{i_{n}}}^{\prime}\right) .
$$

We see that $\psi$ is homotopy equivalent to $f \chi$. Hence, our task is to show that $\chi$ is a quasi-isomorphism. To this end we will use the natural map $r: Y_{\text {Calc }_{\infty}^{0}}(S V) \rightarrow$ $Y_{\mathrm{Calc}^{0}}(S V)$ induced by the map of calculi $\mathrm{Calc}_{\infty}^{0} \rightarrow \mathrm{Calc}^{0}$. It is easy to see that this map is a quasi-isomorphism and that $r \chi$ is an isomorphism, therefore $\chi$ is a quasi-isomorphism, hence the statement.

3.13. The algebra $Y_{\text {Calc }_{\infty}}\left(C^{\bullet}(A)\right)$ and the cyclic complex. To adapt the above arguments to the cyclic case, notice first that one can interpret the cyclic part of Theorem 2.6.1 as follows: there is an $L_{\infty}$ action of the graded Lie algebra $\mathfrak{a}$ (cf. 3.7.4) on the $A_{\infty}$ algebra $C_{\bullet}\left(C^{\bullet}(A)\right)$, and one can form a corresponding $A_{\infty}$ algebra cross product $U(\mathfrak{a}) \ltimes C_{\bullet}\left(C^{\bullet}(A)\right)$. One of the ways to explain this is the following. The $A_{\infty}$ algebra $C_{\bullet}\left(C^{\bullet}(A)\right)$ is quasi-isomorphic to a $\mathrm{dg}$ associative algebra $\mathcal{R}$; the free $\operatorname{dg}$ Lie algebra resolution $\mathcal{L}$ of $\mathfrak{a}$ acts on $\mathcal{R}$ by derivations; form a cross product $U(\mathcal{L}) \ltimes \mathcal{R}$. As a complex, it is quasi-isomorphic to $U(\mathfrak{a}) \otimes C_{\bullet}\left(C^{\bullet}(A)\right)$; from this, one can recover the $A_{\infty}$ structure on the latter. For example, the binary product of the generator of $\mathfrak{a}$ with an element $c \in C_{\bullet}\left(C^{\bullet}(A)\right)$ is $B c$ where $B$ is the cyclic differential.

By Example 3.7.4,

$$
Y_{\text {Calc }}\left(\mathcal{V}^{\bullet}(A)\right) \simeq U(\mathfrak{a}) \ltimes Y\left(\mathcal{V}^{\bullet}(A)\right)
$$

One extends (3.10) to a quasi-isomorphism

$$
U(\mathfrak{a}) \ltimes C_{\bullet}\left(C^{\bullet}(A)\right) \rightarrow Y_{\text {Calc }_{\text {alg }}}\left(C^{\bullet}(A)\right)
$$

One can also construct an $A_{\infty}$ quasi-isomorphism

$$
U(\mathfrak{a}) \ltimes Y_{\text {Calc }_{\text {alg }}^{0}}\left(C^{\bullet}(A)\right) \rightarrow Y_{\text {Calc alg }}\left(C^{\bullet}(A)\right)
$$

Both maps

$$
Y_{\text {Calc }_{\infty}}\left(C^{\bullet}(A)\right) \leftarrow Y_{\text {Calc }_{\infty}}\left(\mathcal{V}^{\bullet}(A)\right) \rightarrow Y_{\text {Calc }}\left(\mathcal{V}^{\bullet}(A)\right)
$$

are quasi-isomorphisms. Thus, we have an $A_{\infty}$ quasi-isomorphism from $U(\mathfrak{a}) \ltimes$ $Y\left(\mathcal{V}^{\bullet}(A)\right)$ to $U(\mathfrak{a}) \ltimes C_{\bullet}\left(C^{\bullet}(A)\right)$. From this it is easy to deduce the statement of Theorem 2.7.1 regarding the cyclic complexes. 


\section{Appendix. Hochschild-Gerstenhaber homology}

4.1. Introductory remarks. Let $\mathcal{A}^{\bullet}$ be a Gerstenhaber algebra (or, more generally, a $G_{\infty}$ algebra). In this section we will construct $H G_{\bullet}\left(\mathcal{A}^{\bullet}\right)$, a new homology functor of $\mathcal{A}^{\bullet}$. It is defined by means of an explicit complex and is a limit of a spectral sequence whose $E_{1}$ term is the Hochschild homology of the graded associative algebra $\mathcal{A}^{\bullet}$. This spectral sequence degenerates at $E_{1}$ in important partial cases. The homology $H G_{\bullet}\left(\mathcal{A}^{\bullet}\right)$ is an associative algebra. When the spectral sequence does degenerate, one can view the associative algebra $H G_{\bullet}\left(\mathcal{A}^{\bullet}\right)$ as a deformation of the graded commutative algebra $H_{\bullet}\left(\mathcal{A}^{\bullet}\right)$ with the shuffle product sh, cf. (2.3).

One can extend the definition of $H G_{\bullet}$ and define the negative cyclic Gerstenhaber homology $H G C_{\bullet}^{-}\left(\mathcal{A}^{\bullet}\right)$. It relates to $H G \bullet$ exactly as the Hochschild homology to the negative cyclic homology.

4.2. Hochschild-Gerstenhaber homology vs Hochschild homology. To define $H G \bullet\left(\mathcal{A}^{\bullet}\right)$, recall the two-colored operad $\mathrm{Calc}^{0}$ and its canonical free resolution $\mathrm{Calc}_{\infty}^{0}$ from 3.6.

Definition 4.2.1. For a $G_{\infty}$ algebra $\mathcal{A}^{\bullet}$, let $H G_{\bullet}\left(\mathcal{A}^{\bullet}\right)$ be the homology of the complex $Y_{\text {Calc }_{\infty}^{0}}\left(\mathcal{A}^{\bullet}\right)$.

Let us start by realizing $Y_{\mathrm{Calc}_{\infty}^{0}}\left(\mathcal{A}^{\bullet}\right)$ as an explicit complex. Recall from 3.1 that a $G_{\infty}$ algebra structure on $\mathcal{A}^{\bullet}$ determines a derivation $\delta$ of the Gerstenhaber algebra $\mathcal{F}^{\bullet}\left(\mathcal{A}^{\bullet}\right)=\wedge^{\bullet} \operatorname{Lie}\left(\mathcal{A}^{\bullet}[1]^{*}\right)$. (Here and in all the computations below, as usual, one has to understand the duals properly; cf. Remark 3.1.1). For an associative augmented dg algebra $Y$, we denote by $\operatorname{Bar}(Y)$ its standard bar (bi)complex which computes $\operatorname{Ext}^{\bullet}(k, k)$.

Proposition 4.2.2. There is a natural isomorphism of complexes

$$
Y_{\operatorname{Calc}_{\infty}^{0}}\left(\mathcal{A}^{\bullet}\right) \rightarrow \operatorname{Bar}\left(Y\left(\wedge^{\bullet} \operatorname{Lie}\left(\mathcal{A}^{\bullet}[1]^{*}\right)\right)\right)
$$

This can be seen directly from the definitions. The right hand side in (4.2.2) stands for the enveloping algebra of the dg Gerstenhaber algebra. It is an associative dg algebra, with the differential induced by $\delta$.

In what follows, $A_{\infty}$ is a Gerstenhaber algebra.

TheOREM 4.2.3. There is a natural spectral sequence converging to $H G \bullet\left(\mathcal{A}^{\bullet}\right)$ for which

$$
E_{1}=E_{2}=H_{\bullet}\left(\mathcal{A}^{\bullet}\right)
$$

the Hochschild homology of the graded algebra $A_{\infty}$.

An important partial case is the following:

Proposition 4.2.4. Let $A_{\infty}$ is a Gerstenhaber algebra on which both operations are zero. Then

$$
H G_{\bullet}\left(\mathcal{A}^{\bullet}\right) \stackrel{\sim}{\rightarrow} H_{\bullet}\left(\mathcal{A}^{\bullet}\right)=C_{\bullet}\left(\mathcal{A}^{\bullet}\right)
$$

Proof of Theorem 4.2.2. Introduce a filtration on the standard complex $Y_{\mathrm{Calc}_{\infty}^{0}}\left(\mathcal{A}^{\bullet}\right)$ : note that $Y\left(\wedge^{\bullet} \operatorname{Lie}\left(\mathcal{A}^{\bullet}[1]^{*}\right)\right)$ is graded by the number of generators $i_{a}$ occurring in a monomial, and let $F_{n}$ consist of all those cochains that annihilate all elements of degree greater than $n$. 
Proposition 4.2.5. The spectral sequence associated to the filtration $F$ has the properties as in Theorem 4.2.3.

Denote

$$
\mathcal{L}\left(\mathcal{A}^{\bullet}\right)=\operatorname{Lie}\left(\mathcal{A}^{\bullet}[1]^{*}\right)
$$

To prove the above Proposition, let us start by reinterpreting $Y\left(\wedge^{\bullet} \mathcal{L}\left(\mathcal{A}^{\bullet}\right)\right)$.

Let $\epsilon$ be a formal parameter of degree -1 and square zero. Consider the dg Lie algebra $\mathcal{L}\left(\mathcal{A}^{\bullet}\right)[\epsilon]$. The universal enveloping algebra $U\left(\mathcal{L}\left(\mathcal{A}^{\bullet}\right)[\epsilon]\right)$ admits a derivation which is characterized by the following. On $U\left(\mathcal{L}\left(\mathcal{A}^{\bullet}\right) \epsilon\right)=\wedge^{\bullet}\left(\mathcal{L}\left(\mathcal{A}^{\bullet}\right)\right)$, this is just the derivation $\delta$ determined by the $G_{\infty}$ structure; and it is the only such derivation which commutes with the derivation induced by $\frac{\partial}{\partial \epsilon}$. Thus, $U\left(\mathcal{L}\left(\mathcal{A}^{\bullet}\right)[\epsilon]\right)$ becomes a dg associative algebra.

LEMMA 4.2.6. There is a natural isomorphism of $d g$ algebras

$$
Y\left(\wedge^{\bullet}\left(\mathcal{L}\left(\mathcal{A}^{\bullet}\right)\right)\right) \rightarrow U\left(\mathcal{L}\left(\mathcal{A}^{\bullet}\right)[\epsilon]\right)
$$

The proof is straightforward.

Now we have an identification

$$
Y_{\operatorname{Calc}_{\infty}^{0}}\left(\mathcal{A}^{\bullet}\right) \simeq \operatorname{Bar}\left(U\left(\mathcal{L}\left(\mathcal{A}^{\bullet}\right)[\epsilon]\right)\right)
$$

Under this identification, the filtration $F$ becomes as follows. Note that $U\left(\mathcal{L}\left(\mathcal{A}^{\bullet}\right)[\epsilon]\right)$ is graded by the number of factors $\epsilon$ in a monomial, and $F_{n}$ consists of cochains annihilating all elements of degree greater than $n$.

In the situation of Proposition 4.2.4, the differential on $U\left(\mathcal{L}\left(\mathcal{A}^{\bullet}\right)[\epsilon]\right)$ is zero. So, to finish the proof of Proposition 4.2.5, and therefore of Theorem 2.7.1, on can go directly to Lemma 4.2.10.

Note that the graded Lie algebra $\mathcal{L}\left(\mathcal{A}^{\bullet}\right)$ possesses a Lie algebra derivation determined by the commutative product on $\mathcal{A}^{\bullet}$ (or, in the general $G_{\infty}$ case, from the $C_{\infty}$ structure on $\mathcal{A}^{\bullet}$ ). Thus $\mathcal{L}\left(\mathcal{A}^{\bullet}\right)$, as well as $\mathcal{L}\left(\mathcal{A}^{\bullet}\right)[\epsilon]$, becomes a dg Lie algebra. One sees easily that the following is true.

LEMma 4.2.7. The first term of the spectral sequence associated to the filtration $F$ is equal to $H^{\bullet}\left(\mathcal{L}\left(\mathcal{A}^{\bullet}\right)[\epsilon]\right)$, the cohomology of the differential graded Lie algebra with trivial coefficients.

LEMMA 4.2.8. In the above spectral sequence $E_{2}=E_{1}$.

To prove this, we need some notation. Let $\mathfrak{g}$ be a dg Lie algebra. By PBW, there is an $\operatorname{ad}(\mathfrak{g})$ - invariant isomorphism $U(\mathfrak{g}) \rightarrow S(\mathfrak{g})$. For any $i$, let $U^{>i}(\mathfrak{g})$ be the pre-image of $S^{>i}(\mathfrak{g})$ under this isomorphism. In particular, $U^{>1}(\mathfrak{g})$ is a $\operatorname{ad}(\mathfrak{g})$ complement of $\mathfrak{g}$ in the augmentation ideal.

The differential on $\mathfrak{g}$ induces a differential on $U(\mathfrak{g})$. Denote this differential by $\delta$. Let $\delta_{1}$ be another derivation of degree one of $U(\mathfrak{g})$. Assume that, for any $X \in \mathfrak{g}$,

$$
\delta X-\delta_{1} X \in U^{>1}(\mathfrak{g})
$$

Since the cohomology $H^{\bullet}(\mathfrak{g})$ is computed by the bar complex $\operatorname{Bar}(U(\mathfrak{g}))$, the new differential $\delta_{1}$ acts on $H^{\bullet}(\mathfrak{g})$.

Lemma 4.2.9. Under the assumption (4.1), the action of $\delta_{1}$ on $H^{\bullet}(\mathfrak{g})$ is trivial.

Proof. Recall how the isomorphism between $H^{\bullet}(\mathfrak{g})$ and the cohomology of the bar complex is constructed. One has two standard resolutions of the trivial $U(\mathfrak{g})$ module $k$ : The bar resolution $\operatorname{Bar}_{n}=U(\mathfrak{g}) \otimes \overline{U(\mathfrak{g})}^{\otimes n}$ and the Koszul resolution 
$K_{n}=U(\mathfrak{g}) \otimes \wedge^{n}(\mathfrak{g})$. One has the standard embedding of complexes $i: K_{\bullet} \rightarrow$ Bar• defined by

$$
f \otimes\left(x_{1} \wedge \ldots \wedge x_{n}\right) \mapsto f \otimes \operatorname{Alt}\left(x_{1} \otimes \ldots \otimes x_{n}\right)
$$

for $f \in U(\mathfrak{g})$ and $x_{i} \in \mathfrak{g}$. By the standard techniques of homological algebra, one can split this embedding and construct a projection $j: B_{\bullet} \rightarrow K_{\bullet}$, so that $j i=1$ and $i j$ is homotopic to the identity as a morphism of complexes of $U(\mathfrak{g})$-modules. It is not difficult to see that one can pick $j$ that annihilates modulo $U^{>0}(\mathfrak{g}) \cdot K_{\bullet}$ all elements $f \otimes \operatorname{Alt}\left(x_{1} \otimes \ldots \otimes x_{n}\right)$ where $f \in U(\mathfrak{g}), x_{1} \in U^{>1}(\mathfrak{g})$, and $x_{i} \in \mathfrak{g}$ for $i>1$. But the differential induced by $\delta_{1}$ sends the image of $i$ precisely to such elements. This shows that $\delta_{1}$ acts by zero on the cohomology of the complex $\operatorname{Hom}_{U(\mathfrak{g})}\left(\operatorname{Bar}_{\bullet}, k\right)$ which computes $H^{\bullet}(\mathfrak{g})$.

It remains to show that

Lemma 4.2.10. For a unital Gerstenhaber algebra $\mathcal{A}^{\bullet}$

$$
H^{\bullet}\left(\mathcal{L}\left(\mathcal{A}^{\bullet}\right)[\epsilon]\right) \simeq H_{\bullet}\left(\mathcal{A}^{\bullet}\right)
$$

Proof. First, $C^{\bullet}\left(\mathcal{L}\left(\mathcal{A}^{\bullet}\right)[\epsilon]\right)$ is isomorphic to $C^{\bullet}\left(\mathcal{L}\left(\mathcal{A}^{\bullet}\right), S^{\bullet}\left(\mathcal{L}\left(\mathcal{A}^{\bullet}\right)\right)^{*}\right)$, the standard cochain complex with coefficients in $S^{\bullet}\left(\mathcal{L}\left(\mathcal{A}^{\bullet}\right)\right)^{*}$. Since $\mathcal{L}\left(\mathcal{A}^{\bullet}\right)$ is free as a graded Lie algebra, the embedding into $C^{\bullet}\left(\mathcal{L}\left(\mathcal{A}^{\bullet}\right), S^{\bullet}\left(\mathcal{L}\left(\mathcal{A}^{\bullet}\right)\right)^{*}\right)$ of the subcomplex

$$
C^{o} \rightarrow \operatorname{Ker}\left(C^{1} \rightarrow C^{2}\right)
$$

is a quasi-isomorphism where

$$
C^{i}=\operatorname{Hom}\left(\wedge^{i}\left(\mathcal{L}\left(\mathcal{A}^{\bullet}\right)\right), S^{\bullet}\left(\mathcal{L}\left(\mathcal{A}^{\bullet}\right)\right)^{*}\right)
$$

Finally, the complex (4.2) can be written explicitly: let, as before, $C_{\bullet}\left(\mathcal{A}^{\bullet}\right)$ be the usual Hochschild complex, and let $C_{\bullet}^{\prime}\left(\mathcal{A}^{\bullet}\right)$ be the same complex equipped with the bar differential $b^{\prime}$, cf. $[\mathbf{L}]$. Then one can identify $C_{0}$ with $C_{\bullet}^{\prime}\left(\mathcal{A}^{\bullet}\right), \operatorname{Ker}\left(C^{1} \rightarrow C^{2}\right)$ with $C_{\bullet}\left(\mathcal{A}^{\bullet}\right)$, and the differential between the two with the map $1-t$ from $[\mathbf{L}]$. The identification is done as follows:

$$
S\left(\mathcal{L}\left(\mathcal{A}^{\bullet}\right)\right) \stackrel{P B W}{\longrightarrow} U\left(\mathcal{L}\left(\mathcal{A}^{\bullet}\right)\right) \simeq T\left(\mathcal{A}^{\bullet}[1]^{*}\right)
$$

therefore

$$
C^{0} \simeq T\left(\mathcal{A}^{\bullet}[1]\right)=C_{\bullet}^{\prime}\left(\mathcal{A}^{\bullet}\right)
$$

and

$$
\operatorname{Ker}\left(C^{1} \rightarrow C^{2}\right) \simeq \mathcal{A}^{\bullet}[1] \otimes T\left(\mathcal{A}^{\bullet}[1]\right) \simeq C_{\bullet}\left(\mathcal{A}^{\bullet}\right)[1]
$$

Since $\mathcal{A}^{\bullet}$ is unital, $C_{\bullet}^{\prime}\left(\mathcal{A}^{\bullet}\right)$ is contractible, and the theorem is proven.

\section{References}

[A] V. Arnold, The cohomology ring of the colored braid group, Mat. Zametki 5 (1969), 227-231.

[BB] A. Beilinson and J. Bernstein, A proof of Jantzen conjectures, Advances in Soviet Mathematics, 16, Part 1 (1993), 1-50.

[BV] J. Boardman, R. Vogt, Homotopy invariant algebraic structures on topological spaces, LNM 347 (1973).

[BFFLS] F. Bayen, M. Flato, C. Fronsdal, A. Lichnerowicz, D. Sternheimer, Deformation Theory and Quantization, Ann. Phys. 111 (1977). p. 61-151

[BNT] P. Bressler, R. Nest, B. Tsygan, Riemann-Roch theorems via deformation quantization I and II, Advances in Mathematics 167 (2002), 1, 1-73.

[CE] H. Cartan, S. Eilenberg, Homological Algebra, Princeton, 1956

[CS] M. Chas, D. Sullivan, String topology, preprint GT/9911159. 
[Co] F. Cohen, The cohomology of $\mathcal{C}_{n+1}$ spaces, $n \geq 0$, Lecture Notes in Math. 533 (1976), 207-351.

[C] A. Connes, Noncommutative Geometry, New York-London, Academic Press, 1994.

[C1] A. Connes, Noncommutative geometry - year 2000, GAFA 2000, special volume, Part II, 481-559.

[CM] A. Connes, H. Moscovici, Cyclic homology and Hopf algebras, Lett. Math. Phys 48 (1999), 97-108.

[CM1] A. Connes, H. Moscovici, Hopf algebras, cyclic homology and the transverse index theorem, Com. Math. Phys. 198 (1998), 199-246.

[CM2] A. Connes, H. Moscovici, The local index formula in noncommutative geometry, GAFA 5 (1995), 174-243.

[D] V. Drinfeld, On quasi-triangular Hopf algebras and on a group that is closely connected with $\operatorname{Gal}(\overline{\mathbb{Q}}) / \mathbb{Q}$, Leningrad J. Math. 2 (1991), 4, 829-860.

[DT] Yu. Daletski, B. Tsygan, Operations on cyclic and Hochschild complexes, Methods Funct. Anal. Topology 5 (1999), 4, 62-86.

[DGT] Yu. Daletski, I. Gelfand and B. Tsygan, On a variant of noncommutative geometry, Soviet Math. Dokl. 40 (1990), 2, 422-426.

[FM] W. Fulton and R. MacPherson, A compactification of the configuration spaces, Ann. Math. 139 (1994), 183-225.

[FT] B. Feigin and B. Tsygan, Additive K-theory, LMN 1289 (1987), 66-220.

[FT3] B. Feigin and B. Tsygan, Additive K-theory and crystalline cohomology, Funct. Anal. and Appl. 19 (1985).

[F] K. Fukaya, Floer homology of Lagrangian foliation and noncommutative mirror symmetry, Kyoto University preprint 98-08.

[F1] K. Fukaya, Deformation theory, homological algebra, and mirror symmetry, Kyoto University preprint, December, 2001.

[G] M. Gerstenhaber, The cohomology structure of an associative ring, Ann. Math. 78 (1963), 267-288.

[GS] M. Gerstenhaber, S. Schack, The shuffle bialgebra and the cohomology of commutative algebras, Journal of Pure and Applied Algebra 70 (1991), 263-272.

[GV] M. Gerstenhaber, A. Voronov, Homotopy G-algebras and moduli space operad, IMRN (1995), 141-153

[G1] E. Getzler, Cartan homotopy formulas and the Gauss-Manin connection in cyclic homology, Israel Math. Conf. Proc., 7, 65-78.

[GJ] E. Getzler, J. Jones, Operads, homotopy algebra and iterated integrals for double loop spaces, preprint hep-th9403055.

[GJ1] E. Getzler and J. Jones, $A_{\infty}$ algebras and the cyclic bar complex, Illinois J. of Math. 34 (1990), 256-283.

[GK] V. Ginzburg and M. Kapranov, Koszul duality for operads, Duke Mathematical Journal 76 (1994), 1, 203-272.

[HJ] C.E. Hood and J.D.S. Jones, Some algebraic properties of cyclic homology groups, K - Theory, 1 (1987), 361-384.

[HKR] G. Hochschild, B. Kostant, and A. Rosenberg, Differential forms on regular affine algebras, Transactions AMS 102 (1962), 383-408.

[Kh] M. Khalkhali, An approach to operations in cyclic homology, Journal of Pure Appl. Alg. 107 (1996), 1, 47-59.

[K1] M. Kontsevich, Operads and motives in deformation quantization, Lett. Math. Phys. 48 (1999), no. 1, 35-72.

[K] M. Kontsevich, Deformation quantization of Poisson manifolds I, preprint QA/9709040.

[KM] M. Kontsevich and Yu. Manin, Gromov-Witten classes, quantum cohomology and enumerative geometry, Comm. Math. Phys. 164 (1994), 525-562.

[KS] M. Kontsevich and Y. Soibelman, Deformations of algebras over operads and Deligne conjecture, EuroConference Moshé Flato 1999, Vol.I (Dijon), 255-307.

[KS1] M. Kontsevich and Y. Soibelman, Deformations of algebras over operads and Deligne conjecture, EuroConference Moshé Flato 2000, Part III (Dijon), Lett. Math. Phys. 56 (2001), 3, 271-294. 
[LS] T. Lada, J.D. Stasheff, Introduction to sh algebras for physicists, International Journal of Theor. Physics 32 (1993), 1087-1103

[L] J.-L. Loday, Cyclic Homology, Springer Verlag, 1993.

[LSV] J.-L. Loday, J. Stasheff, and A. Voronov (ed.), Operads: Proceedings of Renaissance Conferences, Contemporary Mathematics 202, AMS, Providence RI, 1997.

[M] Yu. Manin, Topics in noncommutative Geometry, M. B. Porter Lectures, Princeton University Press, Princeton, NJ, 1991.

[Ma] M. Mata, PhD thesis, Penn State, 1998.

[May] P. May, Infinite loop space theory, Bull. AMS 83 (1977), 4, 456-494.

[MDS] D. McDuff and J. Salamon, J-holomorphic curves and quantum cohomology, AMS, Providence, RI, University Lecture Series, 6, 1994.

[MS] J. McClure and J. Smith, A solution of Deligne's Hochschild cohomology conjecture, Recent Progress in Homotopy Theory (Baltimore, MD, 2000), Contemp. Math. 293, 2002.

[NT] R. Nest, B. Tsygan, On the cohomology ring of an algebra, Advances in Geometry, in: Progress in Mathematics, Birkhäuser, 172 (1997).

[NT1] R. Nest, B. Tsygan, Algebraic Index theorem for families, Adv.Math. 113, 2, pp. 151-205.

[NT2] R. Nest, B. Tsygan, Fukaya type categories for associative algebras, Deformation Theory and Symplectic Geometry, Mathematical Physics Studies, vol. 20, Klüwer Acad. Publ. (1997)

[P] S. Priddy, Koszul resolutions, Trans. AMS 152 (1970), 239-60.

[R] G. Rinehart, Differential forms on general commutative algebras, Trans. AMS 108 (1963), 139-174.

[Sei] P. Seidel, Fukaya categories and deformations, preprint math.SG/0206155

[St] J. Stasheff, Closed string theory, strong homotopy Lie algebras and the operad actions of moduli space, Perspectives in Mathematical Physics, Conf. Proc. Lect. Notes Math. Phys. III, International Press, Cambridge, MA, 1994, 265-288.

[St1] J. Stasheff, Homotopy associativity of H-spaces, I and II, Trans. AMS 108 (1963), 275-312.

[S] D. Sullivan, Infinitesimal computations in topology, Publ. Math. IHES 47 (1977), 269-331.

[T] D. Tamarkin, Another proof of M.Kontsevich formality theorem, preprint QA $/ 9803025$

[T1] D. Tamarkin, Formality of chain operads of small squares, preprint QA/9809164

[TT] D.Tamarkin, B.Tsygan, Cyclic formality and index theorems, EuroConference Moshé Flato 2000 Part II (Dijon), Letters in Mathematical Physics, 56 (2001), 2, 85-97.

[TT1] D.Tamarkin, B.Tsygan, Noncommutative differential calculus, homotopy BV algebras and formality conjectures, Methods Funct. Anal. and Topology 6 (2000), 2, 85-100.

[Ts] B. Tsygan Homology of Lie algebras of matrices over rings and Hochschild homology, Uspekhi Mat. Nauk 38, 2 (1983), 217-218.

[Ts1] B. Tsygan Formality conjectures for chains, Amer. Math. Soc. Transl., 194, 2 (1999), 261-274.

Department of Mathematics, Northwestern University, Evanston, IL 60208, USA 